\title{
Presburger arithmetic with stars, rational subsets of graph groups, and nested zero tests
}

\author{
Christoph Haase \\ University of Oxford \\ United Kingdom \\ Email: christoph.haase@cs.ox.ac.uk
}

\author{
Georg Zetzsche \\ Max Planck Institute for Software Systems (MPI-SWS) \\ Germany \\ Email: georg@mpi-sws.org
}

\begin{abstract}
We study the computational complexity of existential Presburger arithmetic with (possibly nested occurrences of) a Kleene-star operator. In addition to being a natural extension of Presburger arithmetic, our investigation is motivated by two other decision problems.

The first problem is the rational subset membership problem in graph groups. A graph group is an infinite group specified by a finite undirected graph. While a characterisation of graph groups with a decidable rational subset membership problem was given by Lohrey and Steinberg [J. Algebra, 320(2) (2008)], it has been an open problem (i) whether the decidable fragment has elementary complexity and (ii) what is the complexity for each fixed graph group. The second problem is the reachability problem for integer vector addition systems with states and nested zero tests.

We prove that the satisfiability problem for existential Presburger arithmetic with stars is NEXP-complete and that all three problems are polynomially inter-reducible. Moreover, we consider for each problem a variant with a fixed parameter: We fix the starheight in the logic, the group for the membership problem, and the number of distinct zero-tests in the integer vector addition systems. We establish NP-completeness of all problems with fixed parameters.

In particular, this enables us to obtain a complete description of the complexity landscape of the rational subset membership problem for fixed graph groups: If the graph is a clique, the problem is NL-complete. If the graph is a disjoint union of cliques, it is $\mathrm{P}$-complete. If it is a transitive forest (and not a union of cliques), the problem is NP-complete. Otherwise, the problem is undecidable.
\end{abstract}

\section{INTRODUCTION}

Presburger arithmetic is the first-order theory of the natural numbers with addition and order. Shown decidable by Presburger in 1929 [48], Presburger arithmetic has become a standard tool for showing decidability and complexity results in many areas of computer science such as automata theory, database theory, formal verification and knowledge representation; see also [27].

In many domains, both in theory and practice, the existential fragment of Presburger arithmetic is of particular interest for a variety of reasons. With an NP-complete satisfiability problem [8], this fragment is computationally rather lightweight. Furthermore, highly-optimised decision procedures for existential Presburger arithmetic have been developed and integrated into SMT-solvers such as CVC4 [2] and Z3 [13], which in practice enables solving a variety of problems via a reduction into existential Presburger arithmetic. Finally, existential Presburger arithmetic is expressively-complete: A seminal result due to Ginsburg and Spanier [23] established that the sets of integer vectors definable in Presburger arithmetic coincide with the semi-linear sets, which arise as higher-dimensional generalisations of ultimately periodic sets. Since arbitrary semilinear sets are definable in existential Presburger arithmetic, additional quantifiers do not lead to more expressiveness and only contribute succinctness.

Besides Presburger arithmetic, another classical representation for these sets is available in rational expressions over vectors [20], which consist of vectors, unions, (Minkowski) sums, and Kleene stars. Since each of these representations suits certain applications, it is not only natural, but also useful to consider representations that accommodate both rational expressions and existential Presburger arithmetic.

This is one of the reasons why we investigate the computational complexity of $\exists \mathrm{PA}^{*}$, the existential fragment of Presburger arithmetic enriched with a Kleene-star operator (subsequently star operator for brevity). Since existential Presburger arithmetic readily expresses (Minkowski) sums and unions, this encompasses the expressiveness of rational expressions. Given a formula $\phi(\boldsymbol{x})$, the star operator additionally allows for formulas of the form $\phi^{*}(\boldsymbol{x})$ such that $\phi^{*}(\boldsymbol{v})$ holds if there are $\boldsymbol{v}_{1}, \ldots, \boldsymbol{v}_{k} \in \mathbb{N}^{d}$ such that $\boldsymbol{v}=\boldsymbol{v}_{1}+\cdots+\boldsymbol{v}_{k}$ and $\phi\left(\boldsymbol{v}_{i}\right)$ holds for all $1 \leq i \leq k$. Of course, the star-operator may occur in $\phi(\boldsymbol{x})$ itself; we refer to the maximum number of nested star operators occurring in a formula $\phi$ as the star-height of $\phi$. Thus, existential Presburger arithmetic is the fragment of $\exists \mathrm{PA}^{*}$ of star-height 0 . The fragment of $\exists \mathrm{PA}^{*}$ of star-height one was studied by Piskac and Kunčak [46]. They showed NP-completeness of the satisfiability problem, and furthermore natural applications of $\exists \mathrm{PA}^{*}$ to reasoning about multi-sets with cardinality constraints and a class of integer vector addition systems with states with semi-linear transition updates. One of our contributions is to show that $\exists \mathrm{PA}^{*}$ is NEXP-complete in general, and NP-complete for any arbitrary but fixed starheight. While being an interesting result in its own right, our main motivation for studying $\exists \mathrm{PA}^{*}$ emerges from two other problems, the computational complexity of the rational subset membership problem in graph groups and of reachability in integer vector addition systems with nested zero tests. 


\section{Rational subsets of graph groups}

Graph groups (also known as right-angled Artin groups or free partially commutative groups) are infinite groups that are specified by an undirected simple graph. Here, each vertex represents a generator and an edge dictates that a pair of generators commute. This class of groups has received growing attention in the last decades, from computer science as well as from mathematics. In computer science, this is due to their close connection to Mazurkiewicz traces [17, 18, 19] and their prominent role in a general framework for infinite-state systems [9, 12, 56, 57, 58]. In mathematics, graph groups are currently an area of intense investigation because of their rich subgroup structure [53]: The class of virtually special groups, i.e. finite extensions of subgroups of graph groups, recently turned out to encompass an abundant array of groups, namely Coxeter groups [29], one-relator groups with torsion [54], fully residually free groups [54], and fundamental groups of hyperbolic 3-manifolds [33].

In a tradition initiated by Dehn [14] to consider groups together with their decision problems, there has been substantial interest in algorithmic questions for graph groups, such as the word problem [15, 55], solving equations [17, 18, 43], and membership in subgroups [36, 37] and submonoids [36, 41].

A decision problem that been attracting attention in the last decades is the membership problem for rational subsets, i.e. those accepted by a finite automaton (e.g. [36, 41, 42] and [40] for a survey). This is because they naturally generalise subgroups and submonoids, and have been an important tool for solving equations [16] and other problems [3].

An important contribution in this context is a result of Lohrey and Steinberg [41]. It characterises those graphs for which the corresponding graph group has a decidable rational subset membership problem. However, the procedure they provide has non-elementary complexity and it has remained open until now whether there is an elementary one. The precise complexity has been open both in the case (i) where the graph (from the decidable class) is part of the input and (ii) for each fixed group. We denote problem (ii) as RatMP ${ }^{\text {tf }}$.

As we show, this problem is polynomially inter-reducible with satisfiability of $\exists \mathrm{PA}^{*}$. Therefore, our results imply that the problem is NEXP-complete if the graph (and hence group) is part of the input. Moreover, the NP upper bound for fixed star height leads to a complete description of the complexity for each fixed graph group: If the graph is a clique, the problem is NL-complete. If the graph is a disjoint union of at least two cliques, then it is P-complete. If the graph is a transitive forest (and not a disjoint union of cliques), the problem is NP-complete. In all other cases, Lohrey and Steinberg have established undecidability.

\section{Integer VASS with nested zero tests}

Vector addition systems with states (VASS), equivalently known as Petri nets, are a fundamental model of computation. A VASS comprises a finite-state controller with a finite number of counters ranging over the non-negative integers. When taking a transition, counters can be incremented and decremented provided that resulting counter values are all non-negative. VASS find a plethora of applications, primarily for modelling and reasoning about concurrent systems, but also, for example, in formal language theory, logic, process calculi, see e.g. [51] Sec. 5]. While control-state reachability as well as configuration reachability are decidable for VASS, additionally allowing for testing counters for zero along transitions renders both problems undecidable [44]. A decidable extension of VASS with zero tests has been given by Reinhardt [50]. He showed that reachability in VASS extended with nested zero tests (also called hierarchical zero tests) is decidable. Nested zero tests constraint arbitrary zero tests such that the $k$-th counter can only be tested for zero if at the same time the first up to the $(k-1)$-th counters are tested for zero. An alternative proof of Reinhardt's result has been given by Bonnet [7].

Leaving aside decidability issues, one major obstacle in the automated analysis of VASS is the high computational complexity of decidable decision problems: control-state reachability is EXPSPACE-complete [39. 49], and a non-elementary lower bound for the configuration-reachability problem has recently been established [11]. To the best of the authors' knowledge, no dedicated complexity results have been given for VASS with nested zero tests. For overcoming those high computational costs, relaxations of VASS and their extensions have been investigated with the goal of finding computationally more tractable models that can be used to over-approximate reachability sets of VASS and their extensions, for intance allowing counters to range over the integers, the resulting model being commonly known as integer VASS (ZZ-VASS) [28] or blind counter automata [25]. Configuration and a fortiori control-state reachability for $\mathbb{Z}$-VASS are only NP-complete [28]. $\mathbb{Z}$-VASS and related relaxations have successfully been used to enable the scalable analysis of concurrent programs, see e.g. [1, 5, 22]. Furthermore, integer over-approximations of VASS extended with, for instance, affine transformations [6], and ordered and unordered data [30, 31] have also been studied.

A further contribution of this paper is to show that configuration reachability in $\mathbb{Z}$-VASS extended with nested zero tests $\left(\mathbb{Z}-V A S S^{n z}\right)$ is inter-reducible with satisfiability in $\exists \mathrm{PA}^{*}$. As a consequence, we obtain NEXP-completeness of configuration reachability in $\mathbb{Z}$-VASS ${ }^{\mathrm{nz}}$. Furthermore, our reduction preserves fixed-parameter properties, enabling us to show that configuration reachability is NP-complete when fixing the number of distinct zero-tests.

\section{Main technical tools}

We briefly comment on the main ideas and tools that our results rely on. For the NEXP and NP upper bounds for $\exists P^{*}$, just as in [46], we rely on a Carathéodory-type theorem [21] for decomposing semi-linear sets into semi-linear sets whose sets of period vectors have polynomial cardinality. We additionally exploit that this decomposition, together with results on the descriptional complexity of Boolean operations on semi-linear sets [10], also enables us to witness that a linear set is contained in the solutions of $\phi^{*}(\boldsymbol{x})$ by providing only a polynomial number of linear sets contained in the solutions of $\phi(x)$ as a 
certificate. For the NEXP lower bound, we reduce the succinct circuit satisfiability problem to satisfiability of $\exists \mathrm{PA}^{*}$. To this end, we show that one can define $2^{2^{n}}$ in an $\exists \mathrm{PA}^{*}$ formula of size polynomial in $n$. Moreover, we express a universal quantifier ranging over $\left[1,2^{n}\right]$. This allows us to guess an evaluation of the succinct circuit as a number in $\left[1,2^{2^{n}}\right]$ and express consistency with the gates using the quantifier.

In the translation of RatMP ${ }^{\text {tf }}$ to $\exists \mathrm{PA}^{*}$, we avoid the nonelementary blow up as follows. The procedure of Lohrey and Steinberg [41] builds semi-linear sets by alternating two operations: (i) intersections and (ii) building context-free grammars (out of semi-linear representations) and taking their Parikh image. Since Parikh images of context-free grammars may require exponential semi-linear representations [38], this results in non-elementary complexity. Here, we adapt a construction by Verma, Seidl, and Schwentick [52], which builds an existential Presburger formula for a given context-free grammar. Our modification uses the Kleene star to accommodate grammars that have infinite (but semi-linear) sets of productions. For the remaining reductions, we use ad-hoc constructions.

\section{Preliminaries}

\section{A. General notation}

We denote by $\|\cdot\|$ the $\ell_{\infty}$-norm. Given an $m \times n$ integer matrix $A$, as usual $\|A\|_{1, \infty}=\max _{1 \leq i \leq m} \sum_{j=1}^{n}\left|a_{i j}\right|$. Throughout the paper, we interchangeably treat finite sets of vectors $Q \subseteq \mathbb{Z}^{n}$ as matrices (e.g. by lexicographically ordering the elements of $Q$ ), and vice versa.

\section{B. Semi-linear sets}

Let $\boldsymbol{b} \in \mathbb{N}^{n}$ be a base vectors and $P=\left\{\boldsymbol{p}_{1}, \ldots, \boldsymbol{p}_{k}\right\} \subseteq$ $\mathbb{N}^{n}$ be a finite set of period vectors. The linear set $L(\boldsymbol{b}, P)$ generated by $\boldsymbol{b}$ and $P$ is defined as

$$
L(\boldsymbol{b}, P)=\boldsymbol{b}+\left\{\sum_{i=1}^{k} \lambda_{i} \cdot \boldsymbol{p}_{i}: \lambda_{i} \in \mathbb{N}, 1 \leq i \leq k\right\} .
$$

A semi-linear set is a finite union of linear sets. Given a linear set $N=L(\boldsymbol{b}, P)$, we denote by $\|N\|=\max \{\|\boldsymbol{b}\|,\|\boldsymbol{p}\|$ : $\boldsymbol{p} \in P\}$. For a semi-linear set $M=\bigcup_{i \in I} N_{i}$, we denote by $\|M\|=\max \left\{\left\|N_{i}\right\|: i \in I\right\}$. Given $\boldsymbol{v} \in \mathbb{N}^{n}$ and a semi-linear set $M$, deciding whether $\boldsymbol{v} \in M$ is NP-complete [32].

For a linear set $N=L(\boldsymbol{b}, P) \subseteq \mathbb{N}^{n}$, we can a priori only derive $|P| \leq(\|N\|+1)^{n}$ as a bound on the cardinality of $P$. The following paraphrased result due to Eisenbrand and Shmonin shows that $N$ is equivalent to a semi-linear set in which the cardinality of all sets of period vectors is small.

Proposition II.1 (Thm. 1 in [21]). Let $N=L(\boldsymbol{b}, P) \subseteq \mathbb{N}^{n}$ be a linear set. Then $N=\bigcup_{i \in I} L\left(\boldsymbol{b}, P_{i}\right)$ such that $P_{i} \subseteq P$ and $\left|P_{i}\right| \leq 2 n \log (4 n\|N\|)$ for all $i \in I$.

In particular, Proposition II.1 enables us to assume that sets of period vectors of semi-linear sets have small cardinality.

Corollary II.2. Let $M \subseteq \mathbb{N}^{n}$ be a semi-linear set. Then $M=$ $M^{\prime}$ such that $\|M\|=\left\|M^{\prime}\right\|, M^{\prime}=\bigcup_{i \in I} L\left(\boldsymbol{b}_{i}, P_{i}\right)$ and $\left|P_{i}\right| \leq$ $2 n \log (4 n\|M\|)$ for all $i \in I$.
Given a system of linear Diophantine inequalities $\mathcal{S}: A \cdot \boldsymbol{x} \geq$ $\boldsymbol{c}$ for some $m \times n$ integer matrix $A$, denote by $\llbracket \mathcal{S} \rrbracket=\{\boldsymbol{u} \in$ $\left.\mathbb{N}^{n}: A \cdot \boldsymbol{u} \geq \boldsymbol{c}\right\}$ the set of solutions of $\mathcal{S}$. We will use the following bound on the semi-linear representation of the set of solutions of $\mathcal{S}$, which follows from [47] and [10].

Proposition II.3. Let $\mathcal{S}: A \cdot \boldsymbol{x} \geq \boldsymbol{c}$ be a system of linear Diophantine inequalities. Then $M=\llbracket \mathcal{S} \rrbracket=\bigcup_{i \in I} L\left(\boldsymbol{b}_{i}, P\right)$, $\|M\| \leq(n \cdot\|A\|+\|\boldsymbol{c}\|+2)^{m+n}$, and $A \cdot \boldsymbol{b}_{i} \geq \boldsymbol{c}$ for all $i \in I$ and $A \cdot \boldsymbol{p} \geq \mathbf{0}$ for all $\boldsymbol{p} \in P$.

Let $M \subseteq \mathbb{N}^{d}$, the Kleene-star of $M$ is defined as

$$
M^{*}:=\bigcup_{k \geq 0}\left\{\sum_{i=1}^{k} \boldsymbol{v}_{i}: \boldsymbol{v}_{i} \in M\right\} .
$$

Here, the empty sum denotes $\mathbf{0}$. For linear sets, we often write $L^{*}(\boldsymbol{b}, P)$ instead of $(L(\boldsymbol{b}, P))^{*}$.

\section{Presburger arithmetic with stars}

Let $\boldsymbol{x}, \boldsymbol{y}$ be tuples of first-order variables, and let $\boldsymbol{z}=(\boldsymbol{x}, \boldsymbol{y})$ be an $n$-tuple consisting of the first-order variables of $\boldsymbol{x}$ and $\boldsymbol{y}$. A formula of existential Presburger arithmetic of star height 0 is of the form

$$
\phi(\boldsymbol{x})=\exists \boldsymbol{y}: \psi(\boldsymbol{z}),
$$

where $\psi(\boldsymbol{z})$ is a (possibly nested) conjunction and disjunction of linear Diophantine inequalities of the form $\boldsymbol{a} \cdot \boldsymbol{z} \geq c$ for $\boldsymbol{a}^{\top} \in \mathbb{Z}^{n}$ and $c \in \mathbb{Z}$. A formula of existential Presburger arithmetic of star height $k+1$ is of the form of Eq. (1) and additionally allows for atomic formulas $\vartheta^{*}(\boldsymbol{z})$ of star height at most $k$. Existential Presburger arithmetic with stars $\left(\exists \mathrm{PA}^{*}\right)$ is the set of all formulas of existential Presburger arithmetic of star height $k$ for any $k \geq 0$.

The semantics $\llbracket \phi \rrbracket$ of an $\exists \mathrm{PA}^{*}$ formula is given in terms of of subsets of $\mathbb{N}^{d}$ by structural induction. By Proposition II.3 $\llbracket \boldsymbol{a} \cdot \boldsymbol{z} \geq c \rrbracket$ is a semi-linear set, for the remaining cases we define

- $\llbracket \phi(\boldsymbol{z}) \wedge \psi(\boldsymbol{z}) \rrbracket=\llbracket \phi(\boldsymbol{z}) \rrbracket \cap \llbracket \psi(\boldsymbol{z}) \rrbracket$

- $\llbracket \phi(\boldsymbol{z}) \vee \psi(\boldsymbol{z}) \rrbracket=\llbracket \phi(\boldsymbol{z}) \rrbracket \cup \llbracket \psi(\boldsymbol{z}) \rrbracket$

- $\llbracket \vartheta^{*}(\boldsymbol{z}) \rrbracket=\llbracket \vartheta(\boldsymbol{z}) \rrbracket^{*}$

- $\llbracket \exists \boldsymbol{y}: \psi(\boldsymbol{z}) \rrbracket=\pi_{\boldsymbol{x}} \llbracket \psi(\boldsymbol{z}) \rrbracket$, where $\pi_{\boldsymbol{x}}$ denotes the projection onto the variables $\boldsymbol{x}$.

It is not difficult to see, and will more formally be discussed in Section IV that the sets definable in $\exists \mathrm{PA}^{*}$ are semi-linear sets. A formula $\phi(\boldsymbol{x})$ is satisfiable if $\llbracket \phi \rrbracket \neq \emptyset$. (Thus, if $\phi$ has no free variables, then satisfiability means $\llbracket \phi \rrbracket=\{\emptyset\})$.

Remark II.4. We do not allow negation in our formulas in order to avoid complementing semi-linear sets. Atomic formulas of the form $\boldsymbol{a} \cdot \boldsymbol{z} \geq c$ can, however, be negated since $\neg(\boldsymbol{a} \cdot \boldsymbol{z} \geq c) \equiv \boldsymbol{a} \cdot \boldsymbol{z} \leq c-1$.

The length $|\phi|$ of a $\exists \mathrm{PA}^{*}$ formula $\phi$ is defined as the number of symbols required to write down $\phi$, and $\|\phi\|$ denotes the absolute value of the largest constant occurring in $\phi$. Without loss of generality we assume unary encoding of numbers, and that $|\phi| \geq 2$ for any $\phi$. 


\section{Rational subsets of graph groups}

A graph is a pair $(A, I)$, where $A$ is an alphabet and $I \subseteq$ $\mathcal{P}_{2}(A)$. Here, $\mathcal{P}_{k}(S)$ denotes the set of $k$-element subsets of a set $S$. We will also use terminology from Mazurkiewicz traces [19], where $I$ is called an independence relation. For an alphabet $A$, let $A^{-1}=\left\{a^{-1}: a \in A\right\}$ be the alphabet of formal inverses of $A$ and $A^{ \pm 1}=A \cup A^{-1}$. The graph group defined by $(A, I)$, denoted $\mathbb{G}(A, I)$, is the group presented by $\langle a \in A: a b=b a(\{a, b\} \in I)\rangle$. We will work with a definition of $\mathbb{G}(A, I)$ as a monoid as follows. For $u, v \in\left(A^{ \pm 1}\right)^{*}$, let $u \rightarrow v$ if there are words $x, y \in\left(A^{ \pm 1}\right)^{*}$ and a pair $(r, s) \in$ $\{(a b, b a):\{a, b\} \in I)\} \cup\left\{\left(a a^{-1}, \varepsilon\right),\left(a^{-1} a, \varepsilon\right): a \in A\right\}$ such that $u=x r y$ and $v=x s y$. Then, let $\equiv_{A, I}$ be the symmetric, reflexive, transitive closure of $\rightarrow$. We can then define $\mathbb{G}(A, I)=\left(A \cup A^{-1}\right)^{*} / \equiv_{A, I}$. In other words, $\mathbb{G}(A, I)$ consists of $\equiv_{A, I}$ congruence classes $[w]$ for $w \in\left(A^{ \pm 1}\right)^{*}$ that are multiplied by way of $[u][v]=[u v]$. Let $|A|=n$. Observe that if $(A, I)$ is a clique, i.e. $I=\mathcal{P}_{2}(A)$, then $\mathbb{G}(A, I) \cong \mathbb{Z}^{n}$. If $I=\emptyset$, then $\mathbb{G}(A, I)$ is called the free group (over $n$ generators) and is also denoted $F_{n}$.

For a subset $B \subseteq A$, let $I_{B}=I \cap \mathcal{P}_{2}(B)$ and $\pi_{B}:\left(A^{ \pm 1}\right)^{*} \rightarrow$ $\left(B^{ \pm 1}\right)^{*}$ be the morphism with $\pi_{B}(b)=\pi_{B}\left(b^{-1}\right)=b$ for $b \in B$ and $\pi_{B}(a)=\pi_{B}\left(a^{-1}\right)=\varepsilon$ for $a \notin B$. Then $u \equiv_{A, I} v$ implies $\pi_{B}(u) \equiv_{B, I_{B}} \pi_{B}(v)$, so that $\pi_{B}$ induces a morphism $\mathbb{G}(A, I) \rightarrow \mathbb{G}\left(B, I_{B}\right),[u] \mapsto\left[\pi_{B}(u)\right]$, also denoted $\pi_{B}$.

Let $G$ be a group. An automaton over $G$ is a tuple $\mathcal{A}=$ $\left(Q, E, q_{0}, q_{f}\right)$, where $Q$ is a finite set of states, $E \subseteq Q \times G \times Q$ is a finite set of edges, and $q_{0}, q_{f} \in Q$ are its initial and final state, respectively. A configuration of $\mathcal{A}$ is a pair $(p, g) \in$ $Q \times G$ and we write $(p, g) \rightarrow_{\mathcal{A}}\left(p^{\prime}, g^{\prime}\right)$ if there is an edge $\left(p, h, p^{\prime}\right) \in E$ with $g^{\prime}=g h$. Such an automaton describes a subset of $G$, namely $\mathrm{L}(\mathcal{A})=\left\{g \in G:\left(q_{0}, 1\right) \rightarrow_{\mathcal{A}}^{*}\left(q_{f}, g\right)\right\}$, where $\rightarrow_{\mathcal{A}}^{*}$ denotes the reflexive transitive closure of $\rightarrow_{\mathcal{A}}$. The subsets of $G$ of the form $\mathrm{L}(\mathcal{A})$ are called rational subsets.

In algorithms over automata over groups, one usually considers finitely generated (short f.g.) groups, i.e. ones with a finite subset $\Sigma \subseteq G$ so that every element of $G$ can be written as a product of members of $\Sigma$. In that case, the elements of $G$, and hence edge inscriptions, can be encoded by words over $\Sigma$. Since we will work with graph groups, we always assume that automata over $\mathbb{G}(A, I)$ are represented with the generating set $A^{ \pm 1}$ (neither decidability nor complexity of the problems considered here depend on the chosen generating set). The rational subset membership problem for $G$ is the following problem: Given an automaton $\mathcal{A}$ over $G$ and an element $g \in G$, decide whether $g \in \mathrm{L}(\mathcal{A})$.

An important concept in the context of rational subsets of graph groups is that of transitive forests. By a forest, we mean a cycle-free graph where every connected component has a distinguished root vertex. A graph $(A, I)$ is a transitive forest if it can be obtained from a forest by adding an edge between any two nodes that lie on a path between a root and a leaf. The rational subset membership problem for graph groups has been studied by Lohrey and Steinberg [41], who obtained a complete characterisation of those graph groups where the problem is decidable.

Theorem II.5 ([41]). Let $(A, I)$ be a graph. The rational subset membership problem is decidable for $\mathbb{G}(A, I)$ if and only if $(A, I)$ is a transitive forest.

\section{E. Integer VASS with nested zero-tests}

A $d$-dimensional $\mathbb{Z}$-VASS with $k$ nested zero-tests $\left(\mathbb{Z}\right.$-VASS $S_{k}^{\mathrm{nz}}$ for short) is a tuple $V=(Q, Z, E)$, where $Q$ is a finite set of states, $Z \subseteq[0, d]$ is its set of zero tests with $|Z|=k$, and $E \subseteq Q \times[-1,1]^{d} \times Z \times Q$ is its set of edges. A configuration is a pair $(q, \boldsymbol{v}) \in Q \times \mathbb{Z}^{d}$. We write $(q, \boldsymbol{v}) \rightarrow_{V}\left(q^{\prime}, \boldsymbol{v}^{\prime}\right)$ if there is an edge $\left(q, \boldsymbol{u}, \ell, q^{\prime}\right)$ such that (i) $\boldsymbol{v}^{\prime}=\boldsymbol{v}+\boldsymbol{u}$ and (ii) $v_{i}=0$ for every $i \in[0, \ell]$, where $\boldsymbol{v}=\left(v_{1}, \ldots, v_{d}\right)$.

Note that a $\mathbb{Z}$-VASS ${ }_{k}^{\text {zz }}$ can have more than $k$ counters. However, there are at most $k$ elements $\ell$ of $[0, d]$ for which we can check whether all counters $1, \ldots, \ell$ are zero. Moreover, these tests can be performed arbitrarily often during a run. A $\mathbb{Z}$-VASS ${ }^{\mathrm{nz}}$ is a $\mathbb{Z}$-VASS ${ }_{k}^{\mathrm{nz}}$ for some $k \geq 0$.

\section{RESULTS}

In this section, we present the main results of this work. Let us begin with the decision problems we study. The first is the satisfiability problem for $\exists \mathrm{PA}^{*}$ :

Given An $\exists \mathrm{PA}^{*}$ formula $\phi$.

Question Does $\phi$ have a satisfying assignment, i.e. is $\llbracket \phi \rrbracket \neq \emptyset$ ?

Slightly abusing notation, we denote this problem also as $\exists \mathrm{PA}^{*}$. If we restrict the input to $\exists \mathrm{PA}^{*}$ formulas of star-height $k$, then we denote the problem by $\exists \mathrm{PA}_{k}^{*}$.

The second problem concerns rational subsets of graph groups. For the fixed-parameter version, we introduce the branching number of transitive forests. Note that every nonempty transitive forest is either (i) a disjoint union of connected transitive forests, or (ii) has a universal vertex, i.e. a vertex that is adjacent to all other vertices (take the root of the underlying tree). This induces a successive decomposition of the transitive forest into smaller transitive forests: For a disjoint union, take the disjoint connected transitive forests. If there is a universal vertex, remove that vertex to obtain a smaller transitive forest

The decomposition is unique up to isomorphism: This is obvious in the case of a disjoint union. In the case of several universal vertices, note that all possible removals result in isomorphic graphs. This allows us to define the branching number $\beta(A, I)$ of a transitive forest $(A, I)$ : If $A=\emptyset$, then $\beta(A, I)=0$. If $(A, I)$ is a disjoint union of connected transitive forests $\left(A_{1}, I_{1}\right), \ldots,\left(A_{n}, I_{n}\right)$, then $\beta(A, I)=\max \left\{\beta\left(A_{i}, I_{i}\right)\right.$ : $i \in[1, n]\}+(n-1)$. If $(A, I)$ has a universal vertex $u$ and removing $u$ leaves $\left(A^{\prime}, I^{\prime}\right)$, then $\beta(A, I)=\beta\left(A^{\prime}, I^{\prime}\right)$.

The rational subset membership problem for graph groups defined by transitive forests, denoted RatMP ${ }^{\mathrm{tf}}$, is the following:

Given $\mathrm{A}$ transitive forest $(A, I)$, an automaton $\mathcal{A}$ over $\mathbb{G}(A, I)$, and a word $w \in\left(A^{ \pm 1}\right)^{*}$.

Question Does $[w] \in \mathrm{L}(\mathcal{A})$ hold?

If we restrict the input to transitive forests $(A, I)$ with $\beta(A, I) \leq k$, then the problem is denoted RatMP ${ }_{k}^{\text {tf }}$. 
Finally, the reachability problem for $\mathbb{Z}$-VASS $^{\text {nz }}$ is the following:

Given $\mathrm{A} \mathbb{Z}$-VASS ${ }^{\mathrm{nz}} \mathcal{A}$ and configurations $(q, \boldsymbol{v}),(r, \boldsymbol{w})$. Question Does $(q, \boldsymbol{v}) \rightarrow_{\mathcal{A}}^{*}(r, w)$ hold?

If the input is restricted to $\mathbb{Z}$-VASS ${ }^{\mathrm{nz}}$ with $k$ nested zero-tests, we write $\mathbb{Z}$-VASS $S_{k}^{\text {nz }}$. The following is our first main result.

Theorem III.1. The problem $\exists \mathrm{PA}^{*}$ is NEXP-complete and for every fixed $k \in \mathbb{N}, \exists \mathrm{PA}_{k}^{*}$ is NP-complete.

We will prove Theorem III.1 in Section IV. Our second main result is that the problems $\exists \mathrm{PA}^{*}$, RatMP ${ }^{\mathrm{tf}}$ and $\mathbb{Z}$-VASS ${ }^{\mathrm{nz}}$ are polynomial-time inter-reducible.

Theorem III.2. The three problems $\exists \mathrm{PA}^{*}$, RatMP ${ }^{\mathrm{tf}}$ and $\mathbb{Z}$-VASS ${ }^{\mathrm{nz}}$ are polynomially inter-reducible. Moreover, for each fixed $k \in \mathbb{N}$, the reductions translate among $\exists \mathrm{PA}_{k}^{*}$, RatMP ${ }_{k}^{\mathrm{tf}}$, and $\mathbb{Z}$-VASS $S_{k}^{\mathrm{nz}}$.

Theorem III.2 will be shown in Sections Vto VII As obvious consequences, we have:

Corollary III.3. RatMP ${ }^{\mathrm{tf}}$ and $\mathbb{Z}-\mathrm{VASS}^{\mathrm{nz}}$ are NEXP-complete. $\mathrm{RatMP}_{k}^{\mathrm{tf}}$ and $\mathbb{Z}$-VASS ${ }_{k}^{\mathrm{nz}}$ are $\mathrm{NP}$-complete for every $k \in \mathbb{N}$.

In particular, this implies that if we restrict the input of RatMP ${ }^{\text {tf }}$ to a fixed transitive forest $(A, I)$, then the problem is always in NP. With this piece of the puzzle, we can show:

Theorem III.4. Let $(A, I)$ be a graph. Then the rational subset membership problem for $\mathbb{G}(A, I)$ is

1) NL-complete if $(A, I)$ is a clique,

2) $\mathrm{P}$-complete if $(A, I)$ is a disjoint union of at least two cliques,

3) NP-complete if $(A, I)$ is a transitive forest and not a disjoint union of cliques, and

4) undecidable if $(A, I)$ is not a transitive forest.

\section{The COMPlEXITY OF EXISTENTIAL PRESBURGER ARITHMETIC WITH STARS}

This section proves NEXP-completeness of $\exists \mathrm{PA}^{*}$ respectively NP-completeness of $\exists \mathrm{PA}_{k}^{*}$ for $k \geq 0$. We first establish some technical results on semi-linear sets before we provide a bound on the descriptional complexity of the semi-linear representation of the set of solutions of an $\exists \mathrm{PA}^{*}$ formula $\phi$. This bound then gives rise to a decision procedure with the desired upper bounds. We close this section by establishing a matching NEXP lower bound for $\exists P^{*}$.

\section{A. Properties of the Kleene star on semi-linear sets}

As a preparatory step, we analyse the effects on the descriptional complexity of the application of the Kleene star on semi-linear sets. The facts derived below are paraphrased or indirectly stated in [46]; for that reason we only state the results and defer all proofs to the appendix.

The following identities hold for any finite $P, Q \subseteq \mathbb{N}^{d}$ :

$$
\begin{aligned}
& L^{*}(\mathbf{0}, P)+L^{*}(\mathbf{0}, Q)=L^{*}(\mathbf{0}, P \cup Q) \\
& =L(\mathbf{0}, P \cup Q)=L(\mathbf{0}, P)+L(\mathbf{0}, Q)
\end{aligned}
$$

Based on those identities, one can show that semi-linear sets are closed under the star operator.

Lemma IV.1. Let $M=\bigcup_{j \in J} L\left(\boldsymbol{c}_{j}, Q_{j}\right)$ be a semi-linear set. Then $M^{*}=\bigcup_{K \subseteq J} L\left(\boldsymbol{b}_{K}, P_{K}\right)$, where

$$
\begin{array}{rlrl}
\boldsymbol{b}_{K} & :=\sum_{k \in K} \boldsymbol{c}_{k} & C_{K}:=\bigcup_{k \in K}\left\{\boldsymbol{c}_{k}\right\} \\
Q_{K}:=\bigcup_{k \in K} Q_{k} & P_{K}:=C_{K} \cup Q_{K} .
\end{array}
$$

For our purposes, this lemma is too weak: there is no bound on the cardinality of the $P_{K}$, and the magnitude of $\boldsymbol{b}_{K}$ depends on $|J|$, which may be doubly-exponential in $\|M\|$. Building upon Lemma IV.1, we can derive the following lemma whose statement and proof are adapted from [46, Thm. 2].

Lemma IV.2. Let $M=\bigcup_{j \in J} L\left(\boldsymbol{c}_{j}, Q_{j}\right) \subseteq \mathbb{N}^{n}$ be a semi-linear set, and let $c=2 n \log (4 n\|M\|)$. Then $M^{*}=$ $\bigcup_{i \in I} L\left(\boldsymbol{b}_{i}, P_{i}\right)$ such that for every $i \in I$,

- $\boldsymbol{b}_{i}=\sum_{k \in K} \boldsymbol{c}_{k}$ for some $K \subseteq J$ with $|K| \leq c$,

- $P_{i} \subseteq \bigcup_{j \in J}\left\{\boldsymbol{c}_{j}\right\} \cup \bigcup_{k \in K} Q_{k}$ with $\left|P_{i}\right| \leq c$, and

- $\left\|M^{*}\right\| \leq c \cdot\|M\|$.

\section{B. Properties of intersections of linear sets}

We also need a bound on the descriptional complexity of intersecting $k$ linear sets, which are provided by the following lemma. Its proof is analogous to the proof of Theorem 6 in [10] and deferred to the appendix.

Lemma IV.3. Let $M_{j}=L\left(\boldsymbol{c}_{j}, Q_{j}\right) \subseteq \mathbb{N}^{n}$ such that $\left\|M_{j}\right\| \leq$ $s$ and $\left|Q_{j}\right| \leq m, 1 \leq j \leq k$. Then $L:=\bigcap_{1<j<k} M_{j}=$ $\bigcup_{i \in I} L\left(\boldsymbol{b}_{i}, P\right)$ such that

- $\|L\| \leq(k \cdot m \cdot s+1)^{O(k \cdot n)}$

- for every $\boldsymbol{b}_{i}$ and $1 \leq j \leq k$ there is some $\boldsymbol{d} \geq \mathbf{0}$ such that $\boldsymbol{b}_{i}=\boldsymbol{c}_{j}+Q_{j} \cdot \boldsymbol{d}$, and

- for every $1 \leq j \leq k$ there is some non-negative matrix $R$ such that $P=Q_{j} \cdot R$.

\section{Bounds on the semi-linear representation of $\exists \mathrm{PA}^{*}$ solutions}

We are now fully prepared to give an estimation on the descriptional complexity of the semi-linear representation of the set of solutions of an $\exists \mathrm{PA}^{*}$ formula. To this end, we first prove a technical lemma that establishes bounds for conjunctive $\exists \mathrm{PA}^{*}$ formulas in which the maximum constants of the semilinear representation of the top-level $\phi_{i}$ formulas is bounded.

Lemma IV.4. Let $\phi(\boldsymbol{x})=\exists \boldsymbol{y}: \bigwedge_{1<i<j} \phi_{i}(\boldsymbol{x}, \boldsymbol{y}) \wedge$ $\bigwedge_{j<i \leq k} \phi_{i}^{*}(\boldsymbol{x}, \boldsymbol{y})$ be an $\exists \mathrm{PA}^{*}$ formula, $L=\llbracket \bar{\phi} \rrbracket$ and $M_{i}=$ $\llbracket \phi_{i} \rrbracket$ such that $\left\|M_{i}\right\| \leq s$ for all $1 \leq i \leq k$. Then $\|L\| \leq s^{O\left(|\phi|^{3}\right)}$.

Proof. We first estimate $\left\|M_{i}^{*}\right\|$ for $j<i \leq k$. By LemmaIV.2 we have $\left\|M_{i}^{*}\right\| \leq c \cdot s$ with $c \leq 2|\phi| \log (4|\phi| s) \leq O\left(|\phi|^{2} \cdot s\right)$. Hence $\left\|M_{i}^{*}\right\| \leq O\left(|\phi|^{2} \cdot s^{2}\right)$. Likewise, we use Corollary II.2 to derive that the cardinality of the period vectors in the semi-linear representation of $\left\|M_{i}^{*}\right\|$ is bounded by 


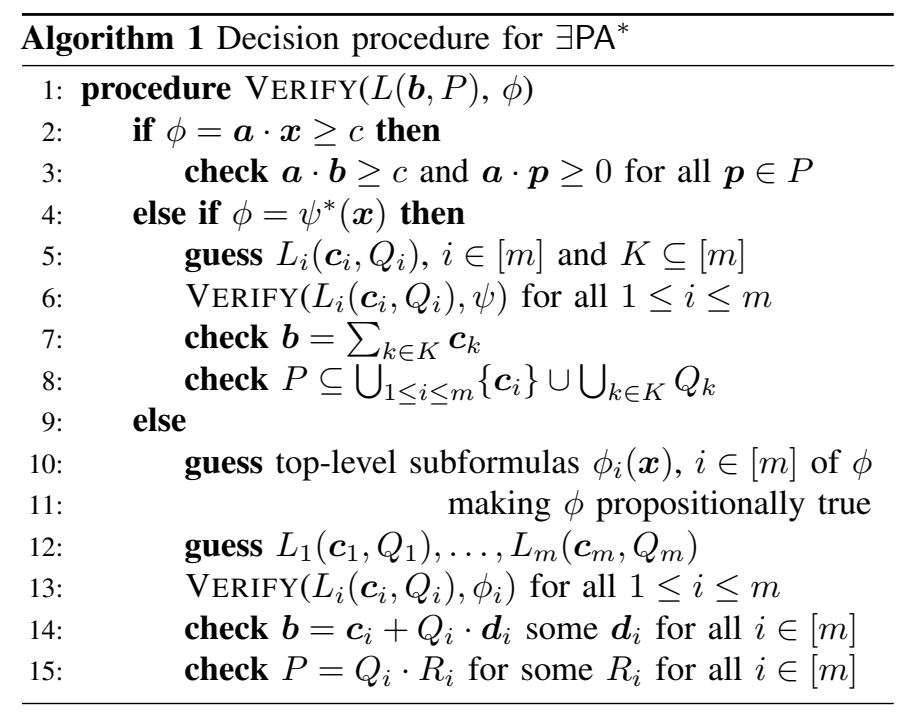

$2|\phi| \log \left(4|\phi|^{3} s^{2}\right) \leq O\left(|\phi|^{2} \cdot s\right)$. Lemma IV.3 implies that for $M:=\bigcap_{1 \leq i \leq j} M_{i} \cap \bigcap_{j<i \leq k} M_{i}^{*}$, we have

$$
\|M\| \leq\left(|\phi| \cdot O\left(|\phi|^{2} \cdot s\right) \cdot O\left(|\phi|^{2} \cdot s^{2}\right)\right)^{O\left(|\phi|^{2}\right)} \leq s^{O\left(|\phi|^{3}\right)} .
$$

The statement follows since $\|L\|=\|M\|$.

As an immediate consequence, we have:

Proposition IV.5. For any $\exists \mathrm{PA}^{*}$ formula $\phi$ of star-height $k$ and $L=\llbracket \phi \rrbracket$, we have $\|L\| \leq\|\phi\|^{|\phi|^{O(k)}}$.

Proof. We show the statement by induction on $k$. The statement follows for $k=0$ from Proposition II.3. For the induction step, apply Lemma IV.4

\section{A decision procedure for $\exists \mathrm{PA}^{*}$}

We now derive one of the main results of this paper:

Proposition IV.6. $\exists \mathrm{PA}^{*}$ is in NEXP, and $\exists \mathrm{PA}_{k}^{*}$ is in NP for any $k \geq 0$.

From Proposition II.3, we know that the constants in the semi-linear representation of the set of solutions of an $\exists \mathrm{PA}^{*}$ formula $\phi(\boldsymbol{x})$ are doubly-exponentially bounded for arbitrary star height, and singly-exponentially bounded for fixed star height. However, checking satisfiability of $\phi$ is not possible by merely guessing some small $\boldsymbol{v} \in \llbracket \phi \rrbracket$. While we can easily verify whether $\boldsymbol{a} \cdot \boldsymbol{v} \geq c$, for subformulas of the form $\psi^{*}(\boldsymbol{x})$ of $\phi$ there is no obvious way to check whether $v \in \llbracket \psi \rrbracket^{*}$.

Instead, the non-deterministic procedure VERIFY (Algorithm 11) proceeds as follows: On input $L(\boldsymbol{b}, P)$ and $\phi$, VERIFY recursively determines whether $L(\boldsymbol{b}, P) \subseteq \llbracket \phi \rrbracket$. The recursion is on the star height of $\phi$. In the base case, $\phi$ is a linear inequality $\boldsymbol{a} \cdot \boldsymbol{x} \geq c$ and according to Proposition II.3, it is sufficient in Line 3 to check whether $\boldsymbol{a} \cdot \boldsymbol{b} \geq c$ and $\boldsymbol{a} \cdot \boldsymbol{p} \geq 0$ for all $\boldsymbol{p} \in P$. In the recursion step, if $\phi=\psi^{*}(\boldsymbol{x})$, the algorithm guesses $m$ linear sets $L_{i}=L\left(\boldsymbol{c}_{i}, Q_{i}\right)$ in Line 5. The magnitude of each $L_{i}$ can be bounded according to Proposition IV.5, and every $\left|Q_{i}\right|$ can also be bounded according to Corollary II.2 The algorithm then verifies in Line 6 whether $L_{i} \subseteq \llbracket \psi \rrbracket$ for every $1 \leq i \leq m$.
If that is the case the algorithm determines $L(\boldsymbol{b}, P) \subseteq \llbracket \psi^{*} \rrbracket$ according to Lemma IV.2 via the previously guessed $L_{i}$. Finally, if $\phi$ is a Boolean combination of subformulas of strictly smaller star height, VERIFY guesses subformulas $\phi_{i}$ that make $\phi$ true when viewing $\phi$ as a formula of propositional logic. Those $\phi_{i}$ could be some $\psi^{*}$ or a linear inequality. As in the previous step, in Line 12 the algorithm attempts to guess linear sets $L_{i}$ such that $L_{i} \subseteq \llbracket \phi_{i} \rrbracket$, and subsequently checks in Lines 14 and 15 whether $L(\boldsymbol{b}, P) \subseteq \bigcap_{1<i \leq m} L_{i}$ via Lemma IV.3

In order to decide whether $\bar{\phi}$ of star height $k$ is satistiable, we guess some $L$ such that $\|L\| \leq\|\phi\|^{|\phi|^{O(k)}}$ and invoke $\operatorname{VERIFY}(L, \phi)$. The depth of the recursion tree of VERIFY is bounded by $O(k)$, and due to Corollary II.2 and Proposition IV.5 in every call VERIFY guesses at most $|\phi|^{O(k)}$ linear sets of magnitude at most $\|\phi\|^{|\phi|^{O(k)}}$. Consequently, VERIFY is an NEXP procedure for arbitrary star height, and an NP procedure for fixed star height.

\section{E. Lower bound}

Here, we show the NEXP lower bound for the satisfiability problem of $\exists \mathrm{PA}^{*}$ via a reduction from succinct circuit satisfiability (SC-SAT), which is known to be NEXP-complete [45].

Before we discuss the reduction, we illustrate a key idea by showing that $\exists \mathrm{PA}^{*}$ formulas may require solutions of doubly exponential magnitude. Suppose $\phi$ is a Presburger formula that defines a single number, i.e., $\phi(x)$ is satisfied for exactly one $x \in \mathbb{N}$. Consider the formula

$$
\begin{gathered}
\phi^{\prime} \equiv \exists y, y^{\prime}, z, z^{\prime}:\left(\phi(x) \wedge y+z=1 \wedge y^{\prime}+z^{\prime}=x \wedge\right. \\
\left.\left(y=1 \rightarrow y^{\prime}=x\right) \wedge\left(z=1 \rightarrow z^{\prime}=x\right)\right)^{*} \wedge y=1 \wedge z=y^{\prime} .
\end{gathered}
$$

The formula under the star has two satisfying assignments: $x$ always has the same value and either $\left(y, y^{\prime}, z, z^{\prime}\right)=(1, x, 0,0)$ or $\left(y, y^{\prime}, z, z^{\prime}\right)=(0,0,1, x)$. Since a satisfying assignment of $\phi^{\prime}$ has to be a sum of such satisfying assignments, imposing $y=1$ means the first case occurred exactly once, thus $y^{\prime}=x$. Moreover, $z=y^{\prime}$ means the second case occurred exactly $y^{\prime}=x$ times, hence $z^{\prime}=x^{2}$. Further, $\phi^{\prime}$ only adds constant (i.e. independent of $\phi$ ) length to $\phi$. Thus, repeating this construction $n$ times, starting with $\phi \equiv(x=2)$, leads to an $\exists \mathrm{PA}^{*}$ formula of size linear in $n$ that has $2^{2^{n}}$ as its only solution.

In order to encode an instance of SC-SAT, we will implement a universal quantifier in $\exists \mathrm{PA}^{*}$, stating that a certain assertion $\phi(x, i)$ holds for all $i$. To this end, we will sum up assignments related to $\phi$, but then we need finer control over which assignments occurred how many times. Moreover, this has to be achieved via conditions on the sums. Here, the following observation will be crucial.

Lemma IV.7. Let $a_{0}, \ldots, a_{m} \in \mathbb{N}$ and $b_{0}, \ldots, b_{m} \in\{0,1\}$ with $\sum_{i=0}^{m} a_{i}=\sum_{i=0}^{m} b_{i}$. Then $\sum_{i=0}^{m} a_{i} 2^{i}=\sum_{i=0}^{m} b_{i} 2^{i}$ if and only if $a_{i}=b_{i}$ for every $i \in[0, m]$.

Proof. Let $X$ be a formal variable and consider the ring $\mathbb{Z}[X]$ of polynomials over the variable $X$ with integer coefficients. In $\mathbb{Z}[X]$, consider the polynomials $p(X)=\sum_{i=0}^{m} a_{i} X^{i}$ and 
$q(X)=\sum_{i=0}^{m} b_{i} X^{i}$. We show that $p(1)=q(1)$ and $p(2)=$ $q(2)$ implies $p(X)=q(X)$. If $a_{i} \geq 2$ for some $i \in[0, m]$, then we modify $p_{0}(X):=p(X)$ to obtain $p_{1}(X)=p_{0}(X)-$ $2 X^{i}+X^{i+1}$. Then, $p_{1}$ has non-negative coefficients and verifies $p_{1}(2)=p_{0}(2)$ and $p_{r}(1)=p_{0}(1)-1$ for every $r$. If we repeat this process, the image at 1 strictly decreases in each step, so the process must terminate, say after $s$ steps. Then the polynomial $p_{s}(x)$ has coefficients in $\{0,1\}$. It satisfies $p_{s}(2)=p(2)$ and $p_{s}(1)=p(1)-s$. This implies $p_{s}(2)=p(2)=q(2)$ and since binary representations are unique, this implies $p_{s}(X)=q(X)$. However, that also means $p(1)-s=p_{s}(1)=q(1)=p(1)$, hence $s=0$ and thus $q(X)=p_{s}(X)=p(X)$.

Note that Lemma IV.7 does not hold without the assumption $b_{0}, \ldots, b_{m} \in\{0,1\}$ : Without it, the lemma would say that a non-zero polynomial in $\mathbb{Z}[X]$ cannot have 1 and 2 as a root, but $(X-1)(X-2)$ is an obvious counter-example to that.

Let us now show how to encode SC-SAT by successively defining predicates. Here, $m, n, k$ are fixed parameters for each formula, which will have size polynomial in $m, n, k$ :

1) We start with some auxiliary predicates expressible in $\exists \mathrm{PA}$. In what follows, we will use $y=\left\langle y_{0}, \ldots, y_{n-1}\right\rangle$ as a shorthand for $y_{0}, \ldots, y_{n-1} \in\{0,1\}$ and $y=\sum_{i=0}^{n-1} y_{i} 2^{i}$. Note that $y=\sum_{i=0}^{n-1} y_{i} 2^{i}$ is easily expressed in an $\exists$ PA formula of size quadratic in $n$. Moreover, recall that there is an $\exists$ PA formula $\mu_{n}$ such that $\mu_{n}(u, v, w)$ states $u, v<2^{n}$ and $w=u \cdot v$ : The latter is equivalent to $u=\left\langle u_{0}, \ldots, u_{n-1}\right\rangle \wedge w=\sum_{i=0}^{n-1} u_{i} \cdot 2^{i} \cdot v$. Here, we can express multiplication with $u_{i}$ because $u_{i} \in\{0,1\}$; and with $2^{i}$ using $i$-fold doubling.

2) Our first predicate in $\exists \mathrm{PA}^{*}$ can define a power $2^{y}$ if $y<2^{n}$ and $y$ is given as bits $y_{0}, \ldots, y_{n-1}$. Formally, $\widehat{\operatorname{pow}}_{n}\left(x, y_{0}, \ldots, y_{n-1}\right)$ means that $y_{0}, \ldots, y_{n-1} \in\{0,1\}$ and $x=2^{\sum_{i=0}^{n-1} y_{i} 2^{i}}$. We define the formula inductively. We can express $\widehat{\operatorname{pow}}_{1}(x)$ just by $x=2$. Once we have a formula for $n-1$, we define one for $n$ :

$$
\begin{aligned}
& y_{0} \in\{0,1\} \wedge \cdots \wedge y_{n-1} \in\{0,1\} \wedge \\
&\left(\widehat{\operatorname{pow}}_{n-1}\left(x^{\prime}, y_{1}^{\prime}, \ldots, y_{n-1}^{\prime}\right) \wedge z=1 \wedge u+v=1\right. \\
&\left.\wedge \bar{u}+\bar{v}=x^{\prime} \wedge\left(u=1 \rightarrow \bar{u}=x^{\prime}\right) \wedge\left(v=1 \rightarrow \bar{v}=x^{\prime}\right)\right)^{*} \\
& \\
& \wedge \bigwedge_{i=1}^{n-1} y_{i}^{\prime}=y_{i} \cdot z \wedge u=1 \wedge v=\bar{u} \wedge x=2^{y_{0}} \cdot \bar{v} .
\end{aligned}
$$

Note that we can easily express $y_{i}^{\prime}=y_{i} \cdot z$ and $x=2^{y_{0}} \cdot \bar{v}$ in Presburger arithmetic because $y_{i} \in\{0,1\}$ for $i \in[0, n-1]$. For the same reason, $y_{i}^{\prime}=y_{i} \cdot z$ implies that in each satisfying assignment of the formula under the star, we have $y_{i}^{\prime}=y_{i}$ for each $i \in[0, n-1]$. The remaining reasoning is similar to our example for $2^{2^{n}}$. Instead of producing $x^{2}$, we use $2^{\sum_{i=0}^{n-1} y_{i} 2^{i}}=\left(2^{\sum_{i=1}^{n-1} y_{i} 2^{i-1}}\right)^{2} \cdot 2^{y_{0}}$.

3) Employing $\widehat{\text { pow }}_{n}$, we can now express powering by a number that is not given in bits, but by a variable. The predicate $\operatorname{pow}_{n}(x, y)$ below holds whenever $y<2^{n}$ and $x=2^{y}$. Here, we can just use $y=\left\langle y_{0}, \ldots, y_{n-1}\right\rangle \wedge \widehat{\text { pow }}_{n}\left(x, y_{0}, \ldots, y_{n-1}\right)$.
4) We now introduce a type of predicates that can be instantiated using other $\exists \mathrm{PA}^{*}$ formulas. It allows us to sum up a sequence of $2^{n}$ tuples, each of which satisfies some given predicate. Moreover, the given predicate has access to a loop variable $i$ (which assumes the values $\left.1, \ldots, 2^{n}\right)$ and to parameters $y_{1}, \ldots, y_{k}<2^{n}$. Suppose $\phi\left(i, x_{1}, \ldots, x_{m}, y_{1}, \ldots, y_{k}\right)$ is an $\exists \mathrm{PA}^{*}$ formula. Then, the predicate $\sum_{i<2^{n}}\left(x_{1}, \ldots, x_{m}\right): \phi\left(i, x_{1}, \ldots, x_{m}, y_{1}, \ldots, y_{k}\right)$ states that $y_{1}, \ldots, y_{k}<2^{n}$ and $x_{j}=\sum_{i=0}^{2^{n}-1} a_{i, j}$ for some numbers $a_{i, j}$ such that $\phi\left(i, a_{i, 1}, \ldots, a_{i, m}, y_{1}, \ldots, y_{k}\right)$ for every $i \in\left[0,2^{n}-1\right]$. The variables before the colon designate which variables are to be added up (namely $x_{1}, \ldots, x_{m}$ ) and which are parameters that should be the same in every summand (namely $y_{1}, \ldots, y_{k}$ ).

$$
\begin{aligned}
& \left(\phi\left(i, x_{1}, \ldots, x_{m}, y_{1}^{\prime}, \ldots, y_{m}^{\prime}\right) \wedge\right. \\
& \left.\bigwedge_{j \in[1, m]} y_{j}^{\prime}=\left\langle y_{j, 1}^{\prime}, \ldots, y_{j, n}^{\prime}\right\rangle \wedge u=1 \wedge v=2^{i}\right)^{*} \wedge \\
& u=2^{n} \wedge v=2^{2^{n}}-1 \wedge \bigwedge_{j \in[1, m]} y_{j}=\left\langle y_{j, 1}, \ldots, y_{j, n}\right\rangle \\
& \wedge \bigwedge_{j \in[1, m], \ell \in[1, n]} y_{j, \ell}^{\prime}=y_{j, \ell} \cdot u .
\end{aligned}
$$

As in $\widehat{\operatorname{pow}}_{n}$, we guarantee that in each satisfying assignment under the star, we have $y_{j, \ell}^{\prime}=y_{j, \ell}$, which in turn means $y_{j}^{\prime}=y_{j}$. This gives the formula under the star access to the parameters $y_{j}$. To see that $i$ assumes each value in $\left[0,2^{n}-1\right]$ exactly once, we use Lemma IV.7 Since we impose $v=$ $2^{2^{n}}-1$ and $u=2^{n}$ outside the star and $2^{2^{n}}-1=\sum_{i=0}^{2^{n}-1} 2^{i}$, the lemma tells us that $v$ has to assume each value $2^{j}$ for $j \in\left[0,2^{n}-1\right]$ exactly once. This implies the constraint on $i$. 5) Using the last step, we can access individual bits of large numbers. bit ${ }_{n}(x, y, z)$ states that $x<2^{2^{n}}, y<2^{n}, z \in\{0,1\}$, and the $y$-th bit in the binary expansion of $x$ is $z$ :

$$
\begin{aligned}
y<2^{n} \wedge z \in\{0,1\} \wedge \sum_{i<2^{n}} x:\left(\left(x=2^{i} \vee x=0\right) \wedge\right. \\
\left.\left(i=y \rightarrow x=z \cdot 2^{i}\right)\right)
\end{aligned}
$$

Here, we say that $x$ has to be a sum of powers of 2 such that the $y$-th power has to occur if and only if $z=1$.

6) Our next predicate multpow $(x, y, z)$ says that $x=2^{y} \cdot z$, $y<2^{n}$, and $z<2^{2^{n}}$.

$$
\begin{aligned}
\sum_{i<2^{2 n}}(x, z):(((i<y \rightarrow x=z=0) \wedge \\
\quad\left(i \geq y \rightarrow \exists b \in\{0,1\}: x=b \cdot 2^{i} \wedge z=b \cdot 2^{i-y}\right)
\end{aligned}
$$

For $x=b \cdot 2^{i}$ and $z=b \cdot 2^{i-y}$, we use the fact that $b \in\{0,1\}$ and pow $_{2 n}$ to define $2^{i}$ and $2^{i-y}$.

7) Let us now express that the binary expansion of $x<2^{2^{m+n}}$ has a one at precisely those positions that are divisible by $2^{n}$. Hence, ones $\mathrm{s}_{n, m}(x)$ says that $x=\sum_{i=0}^{2^{m}-1} 2^{i \cdot 2^{n}}$.

$$
\sum_{i<2^{m}}(x, y):\left(x=2^{i \cdot 2^{n}}\right)
$$


In $x=2^{i \cdot 2^{n}}$, we use the formula $\mu_{n}$ mentioned above and the predicate pow $_{2 n}$.

8) We are now able to state that the bit representation of $x$ is a concatenation of $2^{m}$ copies of the binary representation $y<2^{2^{n}}$. Thus, repeat ${ }_{n, m}(x, y)$ says that $y<2^{2^{n}}$ and $x=$ $\sum_{i=0}^{2^{m}-1} y \cdot 2^{i \cdot 2^{n}}$.

$$
\begin{aligned}
\sum_{i<2^{m}}(x, y):(\exists b \in\{0,1\}, z & <2^{2^{m+n}}: y=b \cdot 2^{i} \\
\wedge \operatorname{ones}_{n, m}(z) & \left.\wedge x=b \cdot 2^{i} \cdot z\right)
\end{aligned}
$$

Note that each summand corresponds to one bit in the binary representation of $y$. Since we sum up shifts of numbers produced by ones ${ }_{n, m}$, that sum consists of $2^{m}$ copies of that representation. Note that we guarantee that the used bits are the ones of $y$ because the sum of the $b \cdot 2^{i}$ has to be $y$ outside of the sum. We can define $2^{i} \cdot z$ using multpow ${ }_{n}$ and then also multiply by $b$ because $b \in\{0,1\}$.

9) We are finally ready to express a (restricted) universal quantifier. Hence, we have again a predicate that is instantiated by another predicate $\phi$. By $\forall^{n, m} i: \phi(x, i)$ we express that $x<2^{2^{n}}$ and for every $i \in\left[0,2^{m}-1\right]$, we have $\phi(x, i)$. We can realise that as follows:

$$
\begin{aligned}
\exists y: \overbrace{\sum_{i<2^{m}} y:\left(\exists z<2^{2^{n}}: \phi(z, i) \wedge y\right.}=2^{\left.i \cdot 2^{n} \cdot z\right)} \\
\wedge \operatorname{repeat}_{n, m}(y, x) .
\end{aligned}
$$

Note that the sum formula $\sigma$ expresses that the binary representation of $y$ is a concatenation of representations of numbers $x_{0}, \ldots, x_{2^{m}-1}$ such that for each $i \in\left[0,2^{m}-1\right]$, we have $\phi\left(x_{i}, i\right)$ and $x_{i}<2^{2^{n}}$. Using repeat r,m $_{n,}$, we then make sure that $x_{0}=\cdots=x_{2^{m}-1}=x$.

Let us now provide a suitable definition of SC-SAT. A circuit $\left(V, v_{0}, C, N\right)$ consists of a finite set $V$ of nodes, a distinguished output node $v_{0} \in V$, and three sets of hyperedges: the conjunctive edges $C \subseteq V \times V \times V$ and the the negation edges $N \subseteq V \times V$. Edges are also called gates. We say that the circuit is satisfiable if there is an assignment $\eta: V \rightarrow\{0,1\}$ such that 1) $\eta\left(v_{0}\right)=1$ and 2) for every $(u, v, w) \in C$, we have $\eta(w)=\min (\eta(u), \eta(v))$ and 3) for every $(u, v) \in N$, we have $\eta(v)=1-\eta(u)$.

A succinct circuit representation $(n, \alpha, \nu)$ consists of a number $n$ (given in unary) and Boolean formulas $\quad \alpha\left(x_{1}, \ldots, x_{n}, y_{1}, \ldots, y_{n}, z_{1}, \ldots, z_{n}\right) \quad$ and $\nu\left(x_{1}, \ldots, x_{n}, y_{1}, \ldots, y_{n}\right)$. The succinct circuit representation $(n, \alpha, \nu)$ describes the circuit $C(n, \alpha, \nu)=\left(V, v_{0}, C, N\right)$, which is defined as follows. We have $V=\{0,1\}^{n}$, $v_{0}=(0, \ldots, 0)$, and $C=\{(x, y, z) \in V \times V \times V: \alpha(x, y, z)\}$ and $N=\{(x, y) \in V \times V: \nu(x, y)\}$. Here, $x, y$, and $z$ is short for $x_{1}, \ldots, x_{n}$ and $y_{1}, \ldots, y_{n}$, and $z_{1}, \ldots, z_{n}$, respectively.

The problem succinct circuit satisfiability (SC-SAT) is defined as follows:

Given A succinct circuit representation $(n, \alpha, \nu)$. Question Is the circuit $C(n, \alpha, \nu)$ satisfiable?
We show that satisfiability of $\exists P^{*}$ is NEXP-hard by reducing SC-SAT, which is NEXP-complete [45]. Suppose $(n, \alpha, \nu)$ is a given succinct circuit. First, we turn $\alpha$ and $\nu$ into formulas $\bar{\alpha}$ and $\bar{\nu}$ in $\exists$ PA so that $\alpha\left(x_{1}, \ldots, x_{n}, y_{1}, \ldots, y_{n}, z_{1}, \ldots, z_{n}\right)$ if and only if $\bar{\alpha}\left(\sum_{i=1}^{n} x_{i} \cdot 2^{i-1}, \sum_{i=1}^{n} y_{i} \cdot 2^{i-1}, \sum_{i=1}^{n} z_{i} \cdot 2^{i-1}\right)$ does not hold; analogously for $\bar{\nu}$. Then the following statement is expressible in an $\exists \mathrm{PA}^{*}$ formula of size polynomial in $n$ :

$$
\begin{aligned}
& \exists x<2^{2^{n}}: \\
& \forall^{n, 3 n} i:( \exists u, v, w<2^{n}: i=u+2^{n} \cdot v+2^{2 n} \cdot w \\
& \wedge \operatorname{bit}_{n}\left(x, u, u^{\prime}\right) \wedge \operatorname{bit}_{n}\left(x, v, v^{\prime}\right) \\
& \wedge \operatorname{bit}_{n}\left(x, w, w^{\prime}\right) \\
&\left.\wedge\left(\bar{\alpha}(u, v, w) \vee w^{\prime}=\min \left(u^{\prime}, v^{\prime}\right)\right)\right) \\
& \wedge \forall^{n, 2 n} i:( \exists u, v<2^{n}: i=u+2^{n} \cdot v \\
& \wedge \operatorname{bit}_{n}\left(x, u, u^{\prime}\right) \wedge \operatorname{bit}_{n}\left(x, v, v^{\prime}\right) \\
&\left.\wedge\left(\bar{\nu}(u, v) \vee v^{\prime}=1-u^{\prime}\right)\right) \\
& \wedge \operatorname{bit}_{n}(x, 0,1)
\end{aligned}
$$

Let us explain why it is equivalent to satisfiability of $C(n, \alpha, \nu)$. We encode the assignment of the $2^{n}$ nodes in $C(n, \alpha, \nu)$ in the number $x<2^{2^{n}}$. In Eqs. (3) to (6), we express that the assignment is consistent with all the conjunctive gates. Note that we cannot directly use a formula of the form $\forall^{n, n} i_{1}: \forall^{n, n} i_{2}: \forall^{n, n} i_{3}: \ldots$, because we would need to use the value of $i_{1}$ (and $i_{2}$ ) in the inner formula (but our quantifier $\forall^{n, m}$ allows only two free variables). Therefore, $i$ ranges over $\left[0,2^{3 n}-1\right]$ and is decomposed into $u, v, w \in\left[0,2^{n}-1\right]$ in Eq. (3). In Eqs. (4) and (5), we extract the bits at the nodes identified by $u, v$ and $w$, and in Eq. (6), we require that if there is a conjunctive gate with input nodes $u, v$ and output node $w$, then the node $w$ carries the conjunction of nodes $u$ and $v$. In Eqs. (7) to (9), we do the same for the negation gates. Finally, in Eq. (10), we state that the output node is indeed assigned value 1 .

\section{TRANSLATING $\exists \mathrm{PA}^{*}$ TO $\mathbb{Z}$-VASS ${ }^{\mathrm{nz}}$}

We now give a polynomial-time reduction from the $\exists \mathrm{PA}^{*}$ satisfiability problem to reachability in $\mathbb{Z}$-VASS ${ }^{\mathrm{nz}}$. In fact, we prove a slightly stronger statement and show that sets of natural numbers definable in $\exists \mathrm{PA}^{*}$ are definable by $\mathbb{Z}$-VASS ${ }^{\mathrm{nz}}$. Given $M \subseteq \mathbb{N}^{d}$, we say that $M$ is $\mathbb{Z}$-VASS ${ }^{\mathrm{nz}}$-definable if there is a $\mathbb{Z}$-VASS ${ }^{\mathrm{nz}} V$ in dimension $m+d$ with designated control states $q, r$ such that $\boldsymbol{v} \in M$ if and only if $(q, \mathbf{0}) \stackrel{*}{\rightarrow}(r,(\mathbf{0}, \boldsymbol{v}))$. We moreover require that $V$ only performs zero-tests on the first $m$ counters.

Proposition V.1. There is a polynomial-time reduction from $\exists \mathrm{PA}^{*}$ to $\mathbb{Z}$-VASS ${ }^{\mathrm{nz}}$, i.e., for any $\exists \mathrm{PA}^{*}$ formula $\phi$, $\phi \rrbracket$ is definable by a $\mathbb{Z}$-VASS ${ }^{\mathrm{nz}} V$. If $\phi$ has star height $k$ then $V$ has $k$ nested zero tests.

We prove Proposition V.1 by structural induction on $\phi$. The idea is inspired by the proof of the coNEXP lower bound for the inclusion problem for $\mathbb{Z}$-VASS [28, Lem. 12]. First, 
by renaming variables if necessary, we may with no loss of generality assume that no existential quantifiers occur in $\phi$.

For the induction base case, let $\phi \equiv \boldsymbol{a} \cdot \boldsymbol{x} \geq c$ be a linear inequality such that $\boldsymbol{a}^{\top}=\left(a_{1}, \ldots, a_{d}\right) \in$ $\mathbb{Z}^{d}$. For $i \in[d]$, define $\boldsymbol{a}_{i}=\left(a_{i}, \boldsymbol{e}_{i}\right) \in \mathbb{Z}^{d+1}$, where $\boldsymbol{e}_{i}$ denotes the $i$-th unit vector in dimension $d$. Consider the $\mathbb{Z}$-VASS ${ }^{\mathrm{nz}}$ depicted

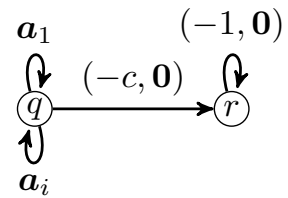

Fig. 1. $\mathbb{Z}-V^{\prime} A S S^{n z}$ defining the solutions of a linear inequality. in Fig. 1. In state $q, V$ has $d$ selfloops adding $\boldsymbol{a}_{i}$ to the counters. Thus $V$ guesses values for $\boldsymbol{x}$ in the last $d$ counters and stores $\boldsymbol{a} \cdot \boldsymbol{x}$ in the first counter. In order to verify $\boldsymbol{a} \cdot \boldsymbol{x} \geq c, V$ removes a value of at least $c$ from the first counter. It follows that $V$ defines $\llbracket \phi \rrbracket$.

In order to deal with Boolean connectives, we first provide a gadget in dimension $3 d$ that checks for $i \in[d]$ whether the contents of the counters $i$ and $d+i$ coincide, and at the same time copies the contents of counter $i$ to counter $2 d+i$. For $i \in[d]$, let $\boldsymbol{v}_{i} \in \mathbb{Z}^{3 d}$ be all zero except for the $i$-th and $(d+i)$-th components which both equal -1 , and the $(2 d+i)$-th component which equals 1 . The $\mathbb{Z}_{\text {-VASS }}{ }^{\text {nz }} C$ depicted in Fig. 2 achieves the desiderata, i.e. $\left(q,\left(\boldsymbol{x}_{1}, \boldsymbol{x}_{2}, \mathbf{0}\right)\right) \stackrel{*}{\rightarrow}(r,(\mathbf{0}, \mathbf{0}, \boldsymbol{x}))$ if and only if $\boldsymbol{x}=\boldsymbol{x}_{1}=\boldsymbol{x}_{2}$.

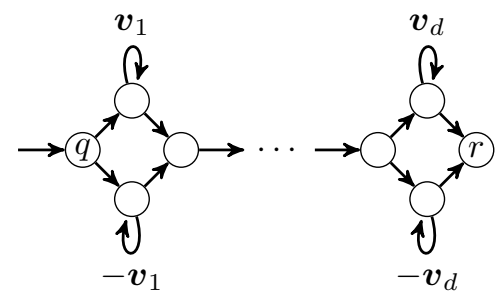

Fig. 2. Gadget $C$ used for handling conjunctions.
For conjunction $\phi \equiv \phi_{1} \wedge \phi_{2}$ let $V_{1}$ and $V_{2}$ be $\quad \mathbb{Z}$-VASS ${ }^{\text {nz }}$ with at most $k \quad$ different zero tests defining $\llbracket \phi_{1} \rrbracket$ and $\llbracket \phi_{2} \rrbracket$ respectively.
Let $\hat{V}_{1}=\left(Q_{1}, Z_{1}, E_{1}\right)$ and $\hat{V}_{2}=\left(Q_{2}, Z_{2}, E_{2}\right)$ be obtained from $V_{1}$ and $V_{2}$ such that $\hat{V}_{1}$ is equivalent to $V_{1}, \hat{V}_{2}$ is equivalent to $V_{2}, Z_{1}=Z_{2},\left|Z_{1}\right|=\left|Z_{2}\right|=k$, and counters not tested for zero in $V_{1}$ and $V_{2}$ do not overlap in $\hat{V}_{1}$ and $\hat{V}_{2}$. The $\mathbb{Z}$-VASS $V$ defining $\llbracket \phi \rrbracket$ now sequentially composes $\hat{V}_{1}$, $\hat{V}_{2}$ and $\hat{C}$. The correctness of the construction is easily seen. Observe that a disjunction $\phi \equiv \phi_{1} \vee \phi_{2}$ can be dealt with analogously, by non-deterministically branching into $\hat{V}_{1}$ and $\hat{V}_{2}$, and by adapting the gadget $C$ appropriately. Note that the construction ensures that any $\exists \mathrm{PA}^{*}$ formula of star height zero is definable by a $\mathbb{Z}$-VASS.

Finally, we provide a construction for formulas of the form $\phi^{*}$. Let $V_{\phi}$ in dimension $m+d$ define $\llbracket \phi \rrbracket$, and let $\hat{V}_{\phi}$ be equivalent to $V$ working in dimension $m+2 d$. Furthermore, for $i \in[d]$ let $\boldsymbol{w}_{i} \in \mathbb{Z}^{m+2 d}$ be all zero except for the $(m+i)$-th component which equals -1 , and the $(m+d+i)$-th component which equals 1 . Then $V$ depicted in Fig. 3 defines $\llbracket \phi^{*} \rrbracket$. First, $V$ traverses $\hat{V}_{\phi}$ thereby pushing some $\boldsymbol{v} \in \llbracket \phi \rrbracket$ onto the counters $[m+1, m+d]$. Next, $V$ adds the contents of the counters $[m+1, m+d]$ to the counters $[m+d+1, m+2 d]$, similar to the gadget $C$ above. When reaching $r, V$ can repeat this

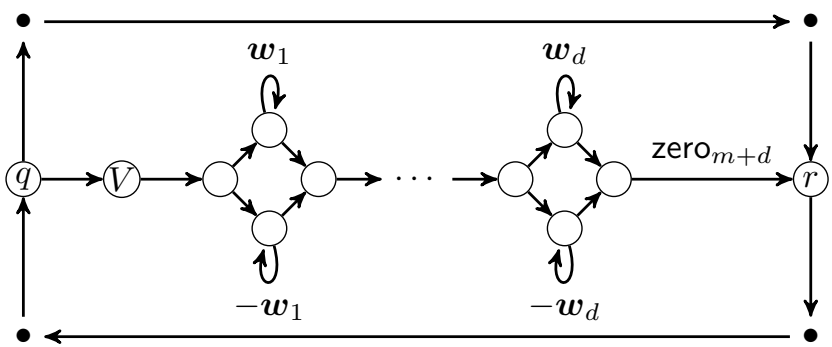

Fig. 3. $\mathbb{Z}$-VASS defining $\llbracket \phi \rrbracket^{*}$, where $\llbracket \phi \rrbracket$ is defined by $V$.

process an arbitrary number of times (and also zero times as required by the definition of the star operator).

Observe that this construction ensures that the $\mathbb{Z}$-VASS ${ }^{\mathrm{nz}}$ $V=(Q, Z, E)$ defining an $\exists \mathrm{PA}^{*}$ formula $\phi$ has $|Z|=k$ if and only if $\phi$ has star height $k$.

\section{TRANSLATING $\mathbb{Z}_{\text {-VASS }}{ }^{\mathrm{nz}}$ TO RatMP ${ }^{\mathrm{tf}}$}

In this section, we show the following.

Proposition VI.1. There is a polynomial reduction from $\mathbb{Z}$-VASS ${ }^{\mathrm{nz}}$ to RatMP ${ }^{\mathrm{tf}}$. Moreover, this reduction maps instances from $\mathbb{Z}$-VASS $S_{k}^{\mathrm{nz}}$ to $\operatorname{RatMP}{ }_{k}^{\mathrm{tf}}$.

We describe the construction, but defer the correctness proof to Appendix $\mathrm{B}$. Let $V=(Q, Z, E)$ be a given $d$-dimensional $\mathbb{Z}$-VASS with $k$ nested zero-tests and let $(q, \boldsymbol{v}),\left(q^{\prime}, \boldsymbol{v}^{\prime}\right)$ be configurations. We may assume that $\boldsymbol{v}=\boldsymbol{v}^{\prime}=\mathbf{0}$. To define the graph group, let $A=\left\{a_{1}, \ldots, a_{d}\right\} \cup\left\{b_{i}: i \in Z\right\}$ and $I=$ $\left\{\left\{a_{i}, b_{j}\right\},\left\{a_{i}, a_{j}\right\} \in \mathcal{P}_{2}(A): i>j\right\}$. This is a transitive forest, because with $I^{\prime}=\left\{\left\{a_{j+1}, b_{j}\right\},\left\{a_{j+1}, a_{j}\right\}: j \in[1, d-1]\right\}$, the graph $\left(A, I^{\prime}\right)$ is a forest that yields $(A, I)$ (see Fig. 4 . Moreover, we have $\beta(A, I)=k$ : If $Z=\left\{m_{1}, \ldots, m_{k}\right\}$, then we can build $(A, I)$ by $m_{1}$ times adding a universal vertex, then a disjoint union with $b_{m_{1}}$, then $m_{2}$ times adding a universal vertex, etc. In the end, we have taken $k$ disjoint unions.

The idea is that since the $a_{i}$ commute pairwise, they generate a group isomorphic to $\mathbb{Z}^{d}$, realises the counters. Since $b_{m_{i}}$ commutes with $a_{m_{i}+1}, \ldots, a_{d}$ but not with $a_{1}, \ldots, a_{m_{i}}$, each generator $b_{m_{i}}$ serves to zero-test counters $a_{1}, \ldots, a_{m_{i}}$ : It cannot be moved past a non-zero product of them.

From $V$, we construct the automaton $\mathcal{A}$ over $\mathbb{G}(A, I)$ as follows. It has states $Q^{\prime}=Q \cup\left\{q_{0}\right\}$ and an edge $\left(q_{0}, b_{i}, q_{0}\right)$ for each $i \in[1, d]$ and one edge $\left(q_{0}, \varepsilon, q\right)$. Moreover, it has an edge $\left(p, b_{\ell}^{-1} a_{1}^{u_{1}} a_{2}^{u_{2}} \cdots a_{d}^{u_{d}}, p^{\prime}\right)$ for each $\left(p,\left(u_{1}, \ldots, u_{d}\right), \ell, p^{\prime}\right) \in E$.

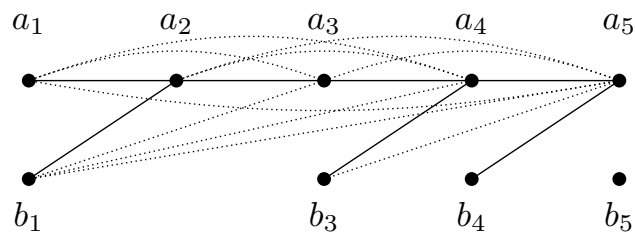

Fig. 4. Independence alphabet used in the proof of Proposition VI.1 in the case of $d=5$ and $Z=\{1,3,4,5\}$. Solid edges are those in $I^{\prime}$, dotted edges are those only in $I$. 


\section{Translating RatMP ${ }^{\text {tf }}$ TO $\exists \mathrm{PA}^{*}$}

In this section, we show the following:

Proposition VII.1. There is a polynomial-time reduction from RatMP $^{\text {tf }}$ to $\exists \mathrm{PA}^{*}$. Moreover, this reduction maps instances of RatMP ${ }_{k}^{\text {tf }}$ to $\exists \mathrm{PA}^{*}$ formulas with star-height $\leq k$.

The central notion in our translation is that of valence automata. What distinguishes them from automata over groups is that they have an input alphabet. This turns out to be convenient for our recursive algorithm.

Let $G$ be a group. A valence automaton over $G$ is a tuple $\mathcal{A}=(Q, \Sigma, E)$, where $Q$ is a finite set of states, $\Sigma$ is an alphabet, and $E \subseteq Q \times G \times \Sigma^{*} \times Q$ is a finite set of edges. A configuration of $\mathcal{A}$ is a triple $(q, g, w) \in Q \times G \times \Sigma^{*}$. The step relation is defined as follows. We have $(q, g, w) \rightarrow_{\mathcal{A}}\left(q^{\prime}, g^{\prime}, w^{\prime}\right)$ if there is an edge $\left(q, h, u, q^{\prime}\right)$ such that $g^{\prime}=g h$ and $w^{\prime}=w u$. This induces a language for each pair of states:

$$
\mathrm{L}(\mathcal{A}, p, q)=\left\{w \in \Sigma^{*}:(p, 1, \varepsilon) \rightarrow_{\mathcal{A}}^{*}(q, 1, w)\right\} .
$$

The non-emptiness problem for a valence automaton asks, given a valence automaton $\mathcal{A}$ and states $p, q \in Q$, whether $\mathrm{L}(\mathcal{A}, p, q) \neq \emptyset$.

As observed in [36], one can reduce the rational subset membership problem for $G$ to the non-emptiness problem of valence automata over $G$ : Given an automaton $\mathcal{A}$ over $G$ and $g \in G$, one can easily construct an automaton $\mathcal{A}_{g}$ over $G$ with $\mathrm{L}\left(\mathcal{A}^{\prime}\right)=\mathrm{L}(\mathcal{A}) g^{-1}$. Then, $1 \in \mathrm{L}\left(\mathcal{A}^{\prime}\right)$ if and only if $g \in \mathrm{L}(\mathcal{A})$. Moreover, we can turn $\mathcal{A}^{\prime}$ into a valence automaton $\mathcal{A}^{\prime \prime}$ by adding a label, say, the letter $a$ to every edge. Then, $\mathrm{L}\left(\mathcal{A}^{\prime \prime}, q_{0}, q_{f}\right) \neq \emptyset$ if and only if $g \in \mathrm{L}(\mathcal{A})$. We will therefore reduce non-emptiness of valence automata to $\exists \mathrm{PA}^{*}$.

Remark VII.2. Note that (unlike, e.g. [56]), we do not use initial and final states in valence automata. This is because we will construct an $\exists \mathrm{PA}^{*}$ formula that describes the Parikh image of a valence automaton for any choice of an initial and a final state. This will allow us to recurse into fewer subinstances in our translation.

Let $\Sigma$ be an alphabet. The Parikh map $\Psi: \Sigma^{*} \rightarrow \mathbb{N}^{\Sigma}$ assigns each word $w \in \Sigma^{*}$ the vector $\Psi(w)$ with $\Psi(w)(a)=|w|_{a}$, where $|w|_{a}$ is the number of occurrences of $a$ in $w$. The Parikh image of a language $L \subseteq \Sigma^{*}$ is $\Psi(L)=\{\Psi(w): w \in L\}$, hence $\Psi(L) \subseteq \mathbb{N}^{\Sigma}$. We will also use a notion of Parikh images of valence automata, which encodes the Parikh image for every choice of initial and final state. For any two states $p, q \in Q$, let $\triangle_{p}^{q}$ be a fresh symbol and let $\Delta=\left\{\triangle_{p}^{q}: p, q \in Q\right\}$. The Parikh image of $\mathcal{A}$, denoted $\Psi(\mathcal{A})$, is defined as $\bigcup_{p, q \in Q} \triangle_{p}^{q}+$ $\Psi\left(\mathrm{L}(\mathcal{A}, p, q)\right.$. Hence, we have $\Psi(\mathcal{A}) \subseteq \mathbb{N}^{\Delta \cup \Sigma}$. Our reduction consists in the following.

Proposition VII.3. Given a transitive forest $(A, I)$ and a valence automaton $\mathcal{A}$ over $\mathbb{G}(A, I)$, one can construct in polynomial time an $\exists \mathrm{PA}^{*}$ formula $\Phi$ with $\llbracket \Phi \rrbracket=\Psi(\mathcal{A})$ and star height at most $\beta(A, I)$.

Note that if this is established, we can reduce non-emptiness of valence automata over $\mathbb{G}(A, I)$ to $\exists \mathrm{PA}^{*}$ : Given a valence automaton $\mathcal{A}$ over $\mathbb{G}(A, I)$, construct an $\exists \mathrm{PA}^{*}$ formula $\Phi$ with $\llbracket \Phi \rrbracket=\Psi(\mathcal{A})$. Then $\mathrm{L}\left(\mathcal{A}, q_{0}, q_{f}\right) \neq \emptyset$ iff the formula $\Phi \wedge \triangle_{q_{0}}^{q_{f}}>0$ is satisfiable. The remainder of this section is devoted to proving Proposition VII.3. The construction is recursive with respect to the decomposition of transitive forests described in Section [III Hence, we need to treat three cases: $(A, I)$ has a universal vertex, $(A, I)$ is a disjoint union, or $(A, I)$ is empty.

Clearly, we may assume that in $\mathcal{A}$, every edge is of the form $(p,[a], x, q)$ with $a \in A^{ \pm 1} \cup\{\varepsilon\}$ and $x \in \Sigma$.

\section{A. Universal vertex}

We first consider the case that the independence alphabet $(A, I)$ has a universal vertex. Suppose there is $u \in A$ such that $\{u, a\} \in I$ for every $a \in A$. Let $A^{\prime}=A \backslash\{u\}$ and $I^{\prime}=I \cap \mathcal{P}_{2}\left(A^{\prime}\right)$. Then the morphism $\mathbb{G}(A, I) \rightarrow \mathbb{G}\left(A^{\prime}, I^{\prime}\right) \times \mathbb{Z}$, $[w] \mapsto\left(\left[\pi_{A^{\prime}}(w)\right],\left[\pi_{\{u\}}(w)\right]\right)$, is an isomorphism. This means we may regard $\mathcal{A}$ as a valence automaton over $\mathbb{G}\left(A^{\prime}, I^{\prime}\right)$ with an additional integer counter. Therefore, we construct a formula for $\Psi(\mathcal{A})$ by first computing a formula for a valence automaton over $\mathbb{G}\left(A^{\prime}, I^{\prime}\right)$ and then imposing the condition that the additional counter be zero at the end.

We construct the valence automaton $\mathcal{A}^{\prime}=\left(Q^{\prime}, \Sigma^{\prime}, E^{\prime}\right)$ over $\mathbb{G}\left(A^{\prime}, I^{\prime}\right)$. We modify $\Sigma$ slightly so as to encode the effect of the additional counter: For each $x \in \Sigma$ and $e \in\{-1,0,1\}$, let $x_{e}$ be a fresh symbol. Recall that all edges in $\mathcal{A}$ are of the form $(p,[a], x, q)$ with $a \in A^{ \pm 1} \cup\{\varepsilon\}$ and $x \in \Sigma$. We obtain $\mathcal{A}^{\prime}$ from $\mathcal{A}$ by replacing 1$)$ each edge of the form $\left(p,\left[u^{e}\right], x, q\right)$, $e \in\{-1,1\}$, with the edge $\left(p, 1, x_{e}, q\right)$ and 2) each edge of the form $(p,[a], x, q), a \in A^{\prime \pm 1} \cup\{\varepsilon\}$, with the edge $\left(p,[a], x_{0}, q\right)$. The alphabet $\Sigma^{\prime}$ of course consists of the symbols $x_{e}$ that occur in these edges. Then, $\mathcal{A}^{\prime}$ is indeed over $\mathbb{G}\left(A^{\prime}, I^{\prime}\right)$.

Suppose $\Phi^{\prime}$ is a formula for $\Psi\left(\mathcal{A}^{\prime}\right)$. Consider the formula

$$
\begin{aligned}
\Phi \equiv \exists\left\{x_{e} \in \Sigma^{\prime}: x \in \Sigma\right\}: & \Phi^{\prime} \wedge \sum_{x_{1} \in \Sigma^{\prime}} x_{1}=\sum_{x_{-1} \in \Sigma^{\prime}} x_{-1} \\
& \wedge \bigwedge_{x \in \Sigma} x=x_{-1}+x_{0}+x_{1} .
\end{aligned}
$$

Slightly abusing notation, if in the sum $x_{-1}+x_{0}+x_{1}$, one of the symbols is not in $\Sigma^{\prime}$, then it is meant not to occur in the sum. Then we clearly have $\llbracket \Phi \rrbracket=\Psi(\mathcal{A})$ :

Step I. Suppose $(A, I)$ has a universal vertex $u \in A$.

1) Construct $\mathcal{A}^{\prime}$ and recursively compute $\Phi^{\prime}$ for $\Psi\left(\mathcal{A}^{\prime}\right)$.

2) Define $\Phi$ as in Eq. (11) and return $\Phi$.

\section{B. Disjoint union}

We now treat the case that $(A, I)$ is a disjoint union of $\left(A_{0}, I_{0}\right)$ and $\left(A_{1}, I_{1}\right)$ (we do not assume that $\left(A_{0}, I_{0}\right)$ and $\left(A_{1}, I_{1}\right)$ are connected).

Modified automata. In order to build the formula for $\mathcal{A}$, we apply our translation recursively to two automata $\mathcal{A}_{0}$ and $\mathcal{A}_{1}$ over $\left(A_{0}, I_{0}\right)$ and $\left(A_{1}, I_{1}\right)$, respectively. For each $p, q \in Q$, let $\square_{p}^{q}$ be a fresh symbol and let $\square=\left\{\square_{p}^{q}: p, q \in Q\right\}$. The automaton $\mathcal{A}_{i}$ is obtained by removing in $\mathcal{A}$ all edges 
labelled with $A_{1-i}$ and then adding, for each $p, q \in Q$, an edge $\left(p, 1, \square_{p}^{q}, q\right)$. Hence, $\square \cup \Sigma$ is the input alphabet for both. Grammars. Let $\mathcal{C}$ be a language class. A $\mathcal{C}$-grammar is a tuple $G=(N, T, P)$, where $N$ is a finite set of nonterminals, $T$ is a finite set of terminals, and $P$ is a finite set of pairs $(B, L)$, where $B \in N$ and $L$ is from $\mathcal{C}$. A pair $(B, L)$ is also denoted $B \rightarrow L$. We write $u \Rightarrow_{G} v$ if there is a pair $(B, L)$ and words $x, y, w \in(N \cup T)^{*}$ such that $u=x B y, v=x w y$ and $w \in L$. Whenever $B \rightarrow L \in P$ and $w \in L$, we say that the pair $B \rightarrow w$ is a production of $G$. If $G$ has finitely many productions, we call it context-free.

The grammar $G_{\mathcal{A}}$ is constructed as follows. It has $Q=\square$ as its set of nonterminals and for every $p, q \in Q$, we have the productions $\square_{p}^{q} \rightarrow \mathrm{L}\left(\mathcal{A}_{i}, p, q\right)$ for $i \in\{0,1\}$.

Proposition VII.4. For every $p, q \in Q$, we have $\mathrm{L}\left(G_{\mathcal{A}}, \square_{p}^{q}\right)=$ $\mathrm{L}(\mathcal{A}, p, q)$.

Presburger formulas. Now that we have expressed the languages of $\mathcal{A}$ in terms of grammars, we can build upon methods for translating grammars into Presburger formulas.

The Parikh image of $G$ is defined as $\Psi(G)=\bigcup_{B \in N} \hat{B}+$ $\Psi(\mathrm{L}(G, B))$. Note that $\Psi(G) \subseteq \mathbb{N}^{\bar{N} \cup T}$. Our construction will build an $\exists \mathrm{PA}^{*}$ formula for $\Psi(G)$ from an $\exists \mathrm{PA}^{*}$ formula describing the right-hand sides in $G$ as follows. The production Parikh image of $G$ is defined as $\Psi \rightarrow(G)=\bigcup_{B \rightarrow L \in P} \bar{B}+\Psi(L)$. Hence, we have $\Psi^{\rightarrow}(G) \subseteq \mathbb{N}^{\bar{N} \cup N \cup T}$.

To build an $\exists \mathrm{PA}^{*}$ formula for the Parikh image of $G$, we extend a construction by Verma, Seidl, and Schwentick [52], which yields an existential Presburger formula for a context-free grammar. We recall the latter in a slightly adapted fashion.

Suppose $G=(N, T, P)$ is context-free with productions $B_{1} \rightarrow w_{1}, \ldots, B_{\ell} \rightarrow w_{\ell}$. For each symbol $x \in N \cup T$, the formula has a variable that counts how often that symbol is produced during a derivation. For each $B \in N$, it also has a variable $\bar{B}$ counting how often $B$ is consumed. It has variables $p_{1}, \ldots, p_{\ell}$ that count how often each production is applied. The variables $\hat{B}$ for $B \in N$ signal which nonterminal is used as the start symbol. In the construction, we use two shorthands: First, $\delta_{B, C}$ stands for 1 if $B$ and $C$ are the same variable (i.e. not only carrying the same value) and 0 otherwise. Second, we use a ternary operator to construct the term $L ? t_{1}: t_{2}$, where $L$ is a literal and $t_{1}$ and $t_{2}$ are ordinary terms. The value of this term is $t_{1}$ if $L$ is true and $t_{2}$ otherwise.

The formula has three conjuncts, $\lambda, \kappa$, and $\chi$. The conjunct $\lambda$ expresses that the number of times each nonterminal is produced and consumed is consistent with the number of applications of each production:

$$
\begin{aligned}
\lambda \equiv \exists p_{1}, \ldots, p_{\ell}: & \bigwedge_{B \in N} \bar{B}=\sum_{i \in J_{B}} p_{i} \\
\wedge & \bigwedge_{x \in N \cup T} x=\sum_{i \in[1, \ell]} \Psi\left(w_{i}\right)(x) \cdot p_{i} \\
& \wedge \bigwedge_{B, C \in N} X_{B, C}=\sum_{i \in J_{B}} \Psi\left(w_{i}\right)(C) \cdot p_{i} .
\end{aligned}
$$

Here, we use $J_{B}=\left\{i \in[1, \ell]: B_{i}=B\right\}$. Moreover, for each $B, C \in N, \lambda$ stores in the variable $X_{B, C}$ whether there is a production with $B$ on the left-hand side and $C$ on the right-hand side. ( $X_{B, C}$ even records the number of such occurrences.)

The formula $\kappa$ selects exactly one of the nonterminals $B$ as the start symbol, i.e. sets $\hat{B}=1$ and $\hat{C}=0$ for every $C \neq B$. Moreover, it requires each nonterminal to be consumed $(\bar{C})$ as many times as it is produced $(C)$. Except for the start symbol, which is consumed once more:

$$
\kappa \equiv \bigvee_{B \in N} \bigwedge_{C \in N} \hat{C}=\delta_{B, C} \wedge \bar{C}=C+\hat{C}
$$

The formula $\chi$ states that every nonterminal is reachable from the start symbol: In the directed graph $\left(N^{\prime}, E\right)$, whose vertices $N^{\prime} \subseteq N$ are the used nonterminals, and with an edge $B \rightarrow C$ if $X_{B, C}>0$, every vertex is reachable from the start symbol. To this end, we assign to every nonterminal $B$ a distance $d_{B}$ so that the start symbol has $d_{B}=0$ and every used nonterminal $B$ is directly reachable from some $C$ with $d_{B}=d_{C}+1$ :

$$
\begin{gathered}
\chi \equiv \exists\left\{d_{B}: B \in N\right\}: \bigwedge_{B \in N}\left(\bar{B}=0 \vee\left(\hat{B}=1 \wedge d_{B}=0\right)\right. \\
\left.\vee\left(\hat{B}=0 \wedge \bigvee_{C \in N} X_{C, B}>0 \wedge d_{B}=d_{C}+1\right)\right) .
\end{gathered}
$$

The complete formula is then $\Phi \equiv \exists_{B, C \in N} X_{B, C} \exists_{B \in N} B: \lambda \wedge$ $\kappa \wedge \chi$. The conditions in $\lambda, \kappa, \chi$ are clearly necessary and Verma, Seidl, and Schwentick [52] have shown sufficiency.

Theorem VII.5 ([52]). If $G$ is context-free as above, then $\Psi(G)=\llbracket \Phi \rrbracket$.

The key observation in our reduction is that using the Kleene star, we can modify $\Phi$ to build an $\exists \mathrm{PA}^{*}$ formula when $G$ is not necessarily context-free, and in particular for $\Psi(\mathcal{A})$. As a first step, note that $\lambda$ is equivalent to the following:

$$
\begin{gathered}
\lambda_{*} \equiv\left(\bigvee_{i \in[1, \ell]}\left(\bigwedge_{j \in[1, \ell]} \bar{B}_{j}=\delta_{i, j} \wedge \bigwedge_{x \in N \cup T} x=\Psi\left(w_{i}\right)(x)\right)\right. \\
\left.\wedge \bigwedge_{B, C \in N} X_{B, C}=(\bar{B}=1) ? C: 0\right)^{*} \cdot
\end{gathered}
$$

This is because each satisfying assignment of the formula under the star assigns variables as $\lambda$ would, if only one production were applied. With the star, we generate all satisfying assignments of $\lambda$. Hence, with $\Phi_{*} \equiv \exists_{B, C \in N} X_{B, C} \exists_{B \in N} B: \lambda_{*} \wedge \kappa \wedge$ $\chi$, we also have $\llbracket \Phi_{*} \rrbracket=\Psi(G)$. Since this formulation does not require a separate variable per production, it can be modified for infinite sets of productions. Suppose $\varphi$ is a formula with $\llbracket \varphi \rrbracket=\Psi \rightarrow(G)$ and consider the formula

$$
\lambda_{*}^{\varphi} \equiv\left(\varphi \wedge \bigwedge_{B, C \in N} X_{B, C}=(\bar{B}=1 ? C: 0)\right)^{*}
$$

and let $\Phi_{\varphi} \equiv \exists_{B, C \in N} X_{B, C} \exists_{B \in N} B: \lambda_{*}^{\varphi} \wedge \kappa \wedge \chi$. Now the following is an easy consequence of Theorem VII.5 
Proposition VII.6. If $\llbracket \varphi \rrbracket=\Psi^{\rightarrow}(G)$, then $\llbracket \Phi_{\varphi} \rrbracket=\Psi(G)$.

Proof. Suppose $\mu \in \llbracket \Phi_{\varphi} \rrbracket$. Then, finitely many satisfying assignments of $\varphi$ justify the satisfaction of $\Phi_{\varphi}$. These satisfying assignments stem from finitely many productions in $G$. Hence, if we build the formula $\Phi_{*}^{\prime}$ for the context-free grammar $G^{\prime}$ that consists of just these productions, then $\mu$ must satisfy $\Phi_{*}^{\prime}$. However, every derivation in $G^{\prime}$ is in particular possible in $G$, which means $\mu \in \Psi(G)$. For the converse, suppose $\mu \in \Psi(G)$. Then $\mu$ stems from a derivation in $G$, which can only involve a finite set of productions. Hence, $\mu \in \Psi\left(G^{\prime}\right)$ for the contextfree grammar $G^{\prime}$ that contains only these productions. If we construct $\Phi_{*}^{\prime}$ as above for $G^{\prime}$, then we have $\mu \in \llbracket \Phi_{*}^{\prime} \rrbracket$. However, since then every satisfying assignment of the $\lambda_{*}$ conjunct in $\Phi_{*}^{\prime}$ also satisfies $\lambda_{*}^{\varphi}$, this implies $\mu \in \llbracket \Phi_{\varphi} \rrbracket$.

We have thus built a formula for $\Psi\left(G_{\mathcal{A}}\right)$ and hence $\Psi(\mathcal{A})$ If we have a formula $\Phi_{i}$ for $\Psi\left(\mathcal{A}_{i}\right)$, then renaming the free variables $\triangle_{p}^{q}$ into $\square_{p}^{q}$ yields formulas $\bar{\Phi}_{i}$ with $\llbracket \bar{\Phi}_{0} \vee \bar{\Phi}_{1} \rrbracket=$ $\Psi^{\rightarrow}\left(G_{\mathcal{A}}\right)$. By Proposition VII.6, we can proceed as follows:

Step II. Let $(A, I)$ be a disjoint union of $\left(A_{0}, I_{0}\right)$ and $\left(A_{1}, I_{1}\right)$.

1) For $i \in\{0,1\}$, compute $\mathcal{A}_{i}$ and recursively compute $\exists \mathrm{PA}^{*}$ formula $\Phi_{i}$ for $\Psi\left(\mathcal{A}_{i}\right)$.

2) For $i \in\{0,1\}$, obtain $\bar{\Phi}_{i}$ from $\Phi_{i}$ by renaming variables $\triangle_{p}^{q}$ to $\bar{\square}_{p}^{q}$ and set $\varphi \equiv \bar{\Phi}_{0} \vee \bar{\Phi}_{1}$.

3) Obtain $\Phi_{\mathcal{A}}$ from $\Phi_{\varphi}$ by renaming variables $\widehat{\square_{p}^{q}}$ to $\triangle_{p}^{q}$.

\section{Empty graph}

If $(A, I)$ is the empty graph, then valence automata over $\mathbb{G}(A, I)$ are just finite automata, for which we can build a Presburger formula using known methods. We construct the context-free grammar $G_{\mathcal{A}}$ with a productions $\square_{p}^{q} \rightarrow x \square_{r}^{q}$ for each edge $(p, x, 1, r)$ and $\square_{p}^{p} \rightarrow \varepsilon$ for every $p \in Q$. Then, we construct the formula $\Phi$ for $G_{\mathcal{A}}$ as in Theorem VII.5

Step III. Suppose $(A, I)$ is empty.

1) Convert $\mathcal{A}$ into context-free grammar $G_{\mathcal{A}}$.

2) Compute Presburger formula for $G_{\mathcal{A}}$ as in Theorem VII.5

A detailed complexity analysis of Steps $[$ to III can be found in Appendix C-A Since before invoking recursion, the automata are expanded in their alphabets (Step I) or set of edges (Step II), we have to show that these remain polynomial during the linear-depth recursion. We argue that the number $n$ of states is never changed, the alphabet size is at most the number of edges, and we add at most $n^{2}$ edges in each step. Hence, every automaton occurring in the algorithm has at most $|A| \cdot n^{2}$ edges more than the initial one.

\section{REMAINING COMPLEXITIES FOR RatMP ${ }^{\text {tf }}$}

In this section, we prove Theorem III.4. The following Lemma is essentially due to Kambites, Silva, and Steinberg, who stated a version about decidability.

Lemma VIII.1 ([36]). For f.g. groups $G$, the following problems are logspace inter-reducible: rational subset membership for $G$ and non-emptiness for valence automata over $G$.
We begin with the case that $(A, I)$ is a clique. Then $\mathbb{G}(A, I) \cong \mathbb{Z}^{|A|}$. NL-hardness is trivially inherited from reachability in directed graphs. For membership in NL, we apply Lemma VIII.1 and observe that a valence automaton over $\mathbb{Z}^{k}$ is a blind $k$-counter automaton, which can be transformed into a 1-reversal-bounded $(k+1)$-counter automaton [34]. Since $k$ is fixed here, reachability is known to be in NL [26].

Now consider the case that $(A, I)$ is a disjoint union of (at least two) cliques. Since $(A, I)$ is not a clique, it contains two non-adjacent vertices. Thus, $F_{2}$ is a subgroup of $\mathbb{G}(A, I)$. Hence the following version of the Chomsky-Schützenberger theorem [4, 35], together with Lemma VIII.1] tells us that the P-hard problem of deciding non-emptiness for contextfree languages (CFL) [24] reduces to membership of rational subsets of $\mathbb{G}(A, I)$.

Lemma VIII.2 ([4, 35]). Given a CFL L, one can construct in logspace a valence automaton over $F_{2}$ that accepts $L$.

For a $\mathrm{P}$ algorithm, it suffices to decide non-emptiness of a valence automaton $\mathcal{A}$ over a disjoint union of cliques. Let $k$ be the size of the largest clique. Observe that Proposition VII.4 tells us that given $\mathcal{A}$, we can construct a $\mathcal{C}$-grammar grammar for $\mathcal{A}$, where $\mathcal{C}$ is the class of languages accepted by valence automata over $\mathbb{Z}^{k}$. As shown above, non-emptiness for $\mathcal{C}$ is in NL. Due to closure under regular intersection, we can even decide in NL whether a given language from $\mathcal{C}$ intersects a given regular language. Under these conditions, one can decide non-emptiness of $\mathcal{C}$-grammars in polynomial time with a simple saturation procedure as for context-free grammars [56, p. 25].

In light of Corollary III.3, the only remaining piece is NPhardness of RatMP ${ }^{\text {tf }}$ in case $(A, I)$ is not a disjoint union of cliques. Observe that the latter is equivalent to saying that the adjacency relation is not transitive. In particular, $(A, I)$ contains the graph $\longrightarrow \longrightarrow$ as an induced subgraph. This means $\mathbb{G}(A, I)$ has $F_{2} \times \mathbb{Z}$ as a subgroup, so that it suffices to show NP-hardness of rational subset membership for $F_{2} \times \mathbb{Z}$.

To this end, we reduce the subset sum problem to non-emptiness of valence automata over $F_{2} \times \mathbb{Z}$. Given $x_{1}, \ldots, x_{n}, y \in \mathbb{N}$ in binary, one readily constructs a context-free grammar for the language $S=\left\{a^{x_{1}}, \varepsilon\right\}$. $\left\{a^{x_{2}}, \varepsilon\right\} \cdots\left\{a^{x_{n}}, \varepsilon\right\} \cdot\left\{b^{y}\right\}$. With Lemma VIII.2 we build a valence automaton $\mathcal{A}$ over $F_{2}$ accepting $S$. From that, one easily obtains a valence automaton $\mathcal{B}$ over $F_{2} \times \mathbb{Z}$ accepting $S^{\prime}=\left\{w \in S:|w|_{a}=|w|_{b}\right\}$. Then the subset sum instance is positive if and only if $S^{\prime} \neq \emptyset$. Thus, non-emptiness of valence automata over $F_{2} \times \mathbb{Z}$ is NP-hard.

\section{ACKNOWLEDGEMENTS}

This research was initiated during the Gregynog 71717 workshop. We would like to thank the organisers Ranko Lazić and Patrick Totzke, as well as the EPSRC for their generous support. We are indebted to Markus Lohrey for fruitful discussions about graph groups, some of which provided key insights that found their way into the present paper. 


\section{REFERENCES}

[1] K. Athanasiou, P. Liu, and T. Wahl. "Unbounded-Thread Program Verification using Thread-State Equations". In: Automated Reasoning, IJCAR. Vol. 9706. Lect. Notes Comp. Sci. Springer, 2016, pp. 516-531.

[2] C. Barrett, C. L. Conway, M. Deters, L. Hadarean, D. Jovanovi'c, T. King, A. Reynolds, and C. Tinelli. "CVC4". In: Computer Aided Verification, CAV. Vol. 6806. Lect. Notes Comp. Sci. Springer, 2011, pp. 171-177.

[3] L. Bartholdi and P. V. Silva. "Rational subsets of groups". In: (2010). arXiv: 1012.1532

[4] J. Berstel. Transductions and Context-Free Languages. Stuttgart: Teubner, 1979.

[5] M. Blondin, A. Finkel, C. Haase, and S. Haddad. "The Logical View on Continuous Petri Nets". In: ACM Trans. Comput. Log. 18.3 (2017), $24: 1-24: 28$

[6] M. Blondin, C. Haase, and F. Mazowiecki. "Affine Extensions of Integer Vector Addition Systems with States". In: Concurrency Theory, CONCUR. Schloss Dagstuhl - Leibniz-Zentrum fuer Informatik, 2018, 14:1-14:17.

[7] R. Bonnet. "Theory of Well-Structured Transition Systems and Extended Vector-Addition Systems". Thèse de doctorat. Laboratoire Spécification et Vérification, ENS Cachan, France, 2013.

[8] I. Borosh and L. B. Treybing. "Bounds on positive integral solutions of linear Diophantine equations". In: Proc. Am. Math. Soc. 55 (1976), pp. 299-304.

[9] P. Buckheister and G. Zetzsche. "Semilinearity and Context-Freeness of Languages Accepted by Valence Automata". In: MFCS. Ed. by K. Chatterjee and J. Sgall. Vol. 8087. Lecture Notes in Computer Science. Berlin/Heidelberg: Springer, 2013, pp. 231-242.

[10] D. Chistikov and C. Haase. "The Taming of the Semi-Linear Set". In: Automata, Languages, and Programming, ICALP. Vol. 55. LIPIcs. Schloss Dagstuhl - Leibniz-Zentrum fuer Informatik, 2016, 128:1128:13. ISBN: 978-3-95977-013-2.

[11] W. Czerwinski, S. Lasota, R. Lazic, J. Leroux, and F. Mazowiecki. "The Reachability Problem for Petri Nets is Not Elementary (Extended Abstract)". In: (2018). arXiv: 1809.07115

[12] E. D'Osualdo, R. Meyer, and G. Zetzsche. "First-order logic with reachability for infinite-state systems". In: Proceedings of the 31st Annual ACM/IEEE Symposium on Logic in Computer Science, LICS '16, New York, NY, USA, July 5-8, 2016. 2016, pp. 457-466.

[13] L. M. de Moura and N. Bjørner. "Z3: An Efficient SMT Solver". In: Tools and Algorithms for the Construction and Analysis of Systems, TACAS. Ed. by C. R. Ramakrishnan and J. Rehof. Vol. 4963. Lecture Notes in Computer Science. Springer, 2008, pp. 337-340.

[14] M. Dehn. "Über unendliche diskontinuierliche Gruppen". In: Mathe matische Annalen 71 (1 1911). In German, pp. 116-144.

[15] V. Diekert. "Word Problems Over Traces which are Solvable in Linear Time”. In: Theoretical Computer Science 74.1 (1990), pp. 3-18.

[16] V. Diekert, C. Gutierrez, and C. Hagenah. "The existential theory of equations with rational constraints in free groups is PSPACE complete". In: Information and Computation 202.2 (2005), pp. 105140. ISSN: 0890-5401.

[17] V. Diekert, A. Jez, and M. Kufleitner. "Solutions of Word Equations Over Partially Commutative Structures". In: 43rd International Colloquium on Automata, Languages, and Programming (ICALP 2016). Vol. 55. Leibniz International Proceedings in Informatics (LIPIcs). Dagstuhl, Germany: Schloss Dagstuhl-Leibniz-Zentrum fuer Informatik, 2016, 127:1-127:14.

[18] V. Diekert and A. Muscholl. "Solvability of Equations in Free Partially Commutative Groups Is decidable". In: Automata, Languages and Programming. Berlin, Heidelberg: Springer Berlin Heidelberg, 2001, pp. 543-554.

[19] V. Diekert and G. Rozenberg, eds. The Book of Traces. Singapore: World Scientific, 1995.

[20] S. Eilenberg and M.-P. Schützenberger. "Rational sets in commutative monoids". In: Journal of Algebra 13.2 (1969), pp. 173-191.

[21] F. Eisenbrand and G. Shmonin. "Carathéodory bounds for integer cones". In: Oper. Res. Lett. 34.5 (2006), pp. 564-568.

[22] J. Esparza, R. Ledesma-Garza, R. Majumdar, P. Meyer, and F. Nikšić "An SMT-Based Approach to Coverability Analysis". In: Computer Aided Verification, CAV. Vol. 8559. Lect. Notes Comp. Sci. Springer, 2014, pp. 603-619.
[23] S. Ginsburg and E. H. Spanier. "Bounded ALGOL-like languages". In: T. Am. Math. Soc. (1964), pp. 333-368.

[24] R. Greenlaw, H. J. Hoover, and W. L. Ruzzo. Limits to Parallel Computation: P-completeness Theory. Oxford University Press, 1995.

[25] S. A. Greibach. "Remarks on blind and partially blind one-way multicounter machines". In: Theoretical Computer Science 7.3 (1978), pp. 311-324.

[26] E. M. Gurari and O. H. Ibarra. "The complexity of decision problems for finite-turn multicounter machines". In: Journal of Computer and System Sciences 22.2 (1981), pp. 220-229.

[27] C. Haase. "A survival guide to Presburger arithmetic". In: SIGLOG News 5.3 (2018), pp. 67-82.

[28] C. Haase and S. Halfon. "Integer Vector Addition Systems with States". In: Reachability Problems - 8th International Workshop, RP 2014, Oxford, UK, September 22-24, 2014. Proceedings. 2014, pp. 112-124.

[29] F. Haglund and D. T. Wise. "Coxeter groups are virtually special". In Advances in Mathematics 224.5 (2010), pp. 1890-1903.

[30] P. Hofman and S. Lasota. "Linear Equations with Ordered Data". In: Concurrency Theory, CONCUR. Schloss Dagstuhl - Leibniz-Zentrum fuer Informatik, 2018, 24:1-24:17.

[31] P. Hofman, J. Leroux, and P. Totzke. "Linear combinations of unordered data vectors". In: Logic in Computer Science, LICS. IEEE Computer Society, 2017, pp. 1-11.

[32] T. Huynh. "The Complexity of Semilinear Sets". In: Elektronische Informationsverarbeitung und Kybernetik 18.6 (1982), pp. 291-338.

[33] I. Agol. "The virtual Haken conjecture. With an appendix by Agol, Daniel Groves, and Jason Manning". In: Documenta Mathematica 18 (2013), pp. 1045-1087.

[34] M. Jantzen and A. Kurganskyy. "Refining the hierarchy of blind multicounter languages and twist-closed trios". In: Information and Computation 185.2 (2003), pp. 159-181.

[35] M. Kambites. "Formal Languages and Groups as Memory". In: Communications in Algebra 37 (1 2009), pp. 193-208.

[36] M. Kambites, P. V. Silva, and B. Steinberg. "On the rational subset problem for groups". In: Journal of Algebra 309 (2007), pp. 622-639.

[37] I. Kapovich, R. Weidmann, and A. G. Myasnikov. "Foldings, Graph of Groups and the Membership Problem". In: International Journal of Algebra and Computation 15.1 (2005), pp. 95-128.

[38] E. Kopczynski and A. W. To. "Parikh Images of Grammars: Complexity and Applications". In: Proceedings of the 25th Annual IEEE Symposium on Logic in Computer Science (LICS 2010). 2010, pp. 80-89.

[39] R. J. Lipton. The Reachability Problem Requires Exponential Space. Tech. rep. 63. Department of Computer Science, Yale University, 1976.

[40] M. Lohrey. "The rational subset membership problem for groups: a survey". In: Groups St Andrews 2013. Ed. by C. M. Campbell, M. R. Quick, E. F. Robertson, and C. M. Roney-Dougal. Vol. 422 London Mathematical Society Lecture Note Series. Cambridge, United Kingdom: Cambridge University Press, 2016, pp. 368-389.

[41] M. Lohrey and B. Steinberg. "The submonoid and rational subset membership problems for graph groups". In: Journal of Algebra 320.2 (2008), pp. 728-755.

[42] M. Lohrey, B. Steinberg, and G. Zetzsche. "Rational subsets and submonoids of wreath products". In: Information and Computation 243 (2015), pp. 191-204.

[43] M. Lohrey and G. Zetzsche. "Knapsack in Graph Groups". In: Theory of Computing Systems 62 (2018), pp. 192-246.

[44] M. L. Minsky. Computation: Finite and Infinite Machines. PrenticeHall, 1967.

[45] C. H. Papadimitriou. Computational Complexity. Addison-Wesley, 1994.

[46] R. Piskac and V. Kuncak. "Linear Arithmetic with Stars". In: Computer Aided Verification, CAV. Vol. 5123. Lect. Notes Comp. Sci. Springer, 2008, pp. 268-280. ISBN: 978-3-540-70543-7.

[47] L. Pottier. "Minimal Solutions of Linear Diophantine Systems: Bounds and Algorithms". In: Rewriting Techniques and Applications, RTA. Vol. 488. Lecture Notes in Computer Science. Springer, 1991, pp. 162 173

[48] M. Presburger. "Über die Vollständigkeit eines gewissen Systems der Arithmetik ganzer Zahlen, in welchem die Addition als einzige Operation hervortritt". In: Comptes Rendus du I congres de Mathematiciens des Pays Slaves. 1929, pp. 92-101.

[49] C. Rackoff. "The Covering and Boundedness Problems for Vector Addition Systems". In: Theor. Comput. Sci. 6 (1978), pp. 223-231. 
[50] K. Reinhardt. "Reachability in Petri Nets with Inhibitor Arcs". In: Electronic Notes in Theoretical Computer Science 223 (2008). Proceedings of the Second Workshop on Reachability Problems in Computational Models (RP 2008), pp. 239-264.

[51] S. Schmitz. "The complexity of reachability in vector addition systems". In: SIGLOG News 3.1 (2016), pp. 4-21.

[52] K. N. Verma, H. Seidl, and T. Schwentick. "On the Complexity of Equational Horn Clauses". In: Proc. of the 20th International Conference on Automated Deduction (CADE-20). 2005, pp. 337-352.

[53] D. Wise. From Riches to Raags: 3-Manifolds, Right-Angled Artin Groups, and Cubical Geometry. American Mathematical Society, 2012.

[54] D. T. Wise. "Research announcement: the structure of groups with a quasiconvex hierarchy". In: Electronic Research Announcements in Mathematical Sciences 16 (2009), pp. 44-55.

[55] C. Wrathall. "Trace monoids with some invertible generators: two decision problems". In: Discrete Applied Mathematics 32.2 (1991), pp. 211-222.

[56] G. Zetzsche. "Monoids as Storage Mechanisms". PhD thesis. Technische Universität Kaiserslautern, 2016

[57] G. Zetzsche. "Silent Transitions in Automata with Storage". In: Proc of the 40th International Colloquium on Automata, Languages and Programming (ICALP 2013). Ed. by F. V. Fomin, R. Freivalds, M Kwiatkowska, and D. Peleg. Vol. 7966. LNCS. Berlin Heidelberg: Springer, 2013, pp. 434-445.

[58] G. Zetzsche. "The Emptiness Problem for Valence Automata or Another Decidable Extension of Petri Nets". In: Proc. of the 9th International Workshop on Reachability Problems (RP 2015). Ed. by M. Bojańczyk, S. Lasota, and I. Potapov. Vol. 9328. LNCS. Berlin Heidelberg: Springer, 2015, pp. 166-178.

\section{APPENDIX A}

\section{MISSING PROOFS FROM SECTION IV}

Lemma IV.1. Let $M=\bigcup_{j \in J} L\left(\boldsymbol{c}_{j}, Q_{j}\right)$ be a semi-linear set. Then $M^{*}=\bigcup_{K \subseteq J} L\left(\boldsymbol{b}_{K}, P_{K}\right)$, where

$$
\begin{aligned}
\boldsymbol{b}_{K} & :=\sum_{k \in K} \boldsymbol{c}_{k} & C_{K} & :=\bigcup_{k \in K}\left\{\boldsymbol{c}_{k}\right\} \\
Q_{K} & :=\bigcup_{k \in K} Q_{k} & P_{K} & :=C_{K} \cup Q_{K} .
\end{aligned}
$$

Proof. Let $\boldsymbol{v} \in M^{*}$, by grouping and summing up vectors according to the linear set they originate from, we can write $\boldsymbol{v}=\boldsymbol{v}_{1}+\cdots+\boldsymbol{v}_{k}$ such that $\boldsymbol{v}_{i} \in L^{*}\left(\boldsymbol{c}_{j_{i}}, Q_{j_{i}}\right)$ for all $1 \leq i \leq k$ and indices $j_{i} \in J$. Observe that for a fixed $i$, we have

$$
\boldsymbol{v}_{i} \in \boldsymbol{c}_{j_{i}}+L^{*}\left(\mathbf{0},\left\{\boldsymbol{c}_{j_{i}}\right\} \cup Q_{j_{i}}\right)=c_{j_{i}}+L\left(\mathbf{0},\left\{\boldsymbol{c}_{j_{i}}\right\} \cup Q_{j_{i}}\right),
$$

where the last equality follows from Eq. (2). Hence

$$
\begin{aligned}
\boldsymbol{v} & \in \sum_{i=1}^{k}\left(\boldsymbol{c}_{j_{i}}+L\left(\mathbf{0},\left\{\boldsymbol{c}_{j_{i}}\right\} \cup Q_{j_{i}}\right)\right) \\
& =\sum_{i=1}^{k} \boldsymbol{c}_{i}+L\left(\mathbf{0}, C_{K} \cup Q_{K}\right) \\
& =L\left(\sum_{i=1}^{k} \boldsymbol{c}_{j_{i}}, C_{K} \cup Q_{K}\right) \\
& =L\left(\boldsymbol{b}_{K}, P_{K}\right),
\end{aligned}
$$

where $K:=\left\{j_{i}: 1 \leq i \leq k\right\}$.

Conversely, suppose $\boldsymbol{v} \in L\left(\boldsymbol{b}_{K}, P_{K}\right)$ for some $K=$ $\left\{j_{1}, \ldots, j_{k}\right\}$. Then there are $\lambda_{1}, \ldots, \lambda_{k}>0$ and $\gamma_{1}, \ldots, \gamma_{k} \geq$ $\mathbf{0}$ such that

$$
\boldsymbol{v}=\sum_{i=1}^{k}\left(\lambda_{i} \cdot \boldsymbol{c}_{j_{i}}+Q_{j_{i}} \cdot \gamma_{i}\right) \in \sum_{i=1}^{k} \lambda_{i} \cdot L\left(\boldsymbol{c}_{j_{i}}, Q_{j_{i}}\right) \subseteq M^{*}
$$

Lemma IV.2. Let $M=\bigcup_{j \in J} L\left(\boldsymbol{c}_{j}, Q_{j}\right) \subseteq \mathbb{N}^{n}$ be a semi-linear set, and let $c=2 n \log (4 n\|M\|)$. Then $M^{*}=$ $\bigcup_{i \in I} L\left(\boldsymbol{b}_{i}, P_{i}\right)$ such that for every $i \in I$,

- $\boldsymbol{b}_{i}=\sum_{k \in K} \boldsymbol{c}_{k}$ for some $K \subseteq J$ with $|K| \leq c$,

- $P_{i} \subseteq \bigcup_{j \in J}\left\{\boldsymbol{c}_{j}\right\} \cup \bigcup_{k \in K} Q_{k}$ with $\left|P_{i}\right| \leq c$, and

- $\left\|M^{*}\right\| \leq c \cdot\|M\|$.

Proof. By Lemma IV.1, we have $M^{*}=\bigcup_{K \subseteq J} L\left(\boldsymbol{d}_{K}, R_{K}\right)$ such that $\boldsymbol{d}_{K}=\sum_{k \in K} \boldsymbol{c}_{k}$ and $R_{K}=C_{K} \cup Q_{K}$, where $C_{K}=$ $\bigcup_{k \in K} c_{k}$ and $Q_{K}=\bigcup_{k \in K}$. By Proposition II.1. for a fixed $K \subseteq J$ we have

$$
L\left(\boldsymbol{d}_{K}, R_{K}\right)=\bigcup_{h \in H_{K}} L\left(\boldsymbol{d}_{K}, S_{h}\right)
$$

such that $S_{h} \subseteq Q_{K}$ and $\left|S_{h}\right| \leq c$ for all $h \in H_{K}$. For $h \in H_{K}$, let $K\left(S_{h}\right)=\left\{k \in K: S_{h} \cap Q_{K} \neq \emptyset\right\}$ and $\boldsymbol{b}_{h}=\sum_{k \in K\left(S_{h}\right)} \boldsymbol{c}_{k}$ We have $\boldsymbol{d}_{K} \in L\left(\boldsymbol{b}_{h}, C_{K}\right)$, and hence

$$
L\left(\boldsymbol{d}_{K}, R_{K}\right) \subseteq \bigcup_{h \in H_{K}} L\left(\boldsymbol{b}_{h}, S_{h} \cup C_{K}\right) \subseteq M^{*} .
$$

Applying Proposition II.1 once more to every $S_{h} \cup C_{K}$, the statement follows.

Lemma IV.3. Let $M_{j}=L\left(\boldsymbol{c}_{j}, Q_{j}\right) \subseteq \mathbb{N}^{n}$ such that $\left\|M_{j}\right\| \leq$ $s$ and $\left|Q_{j}\right| \leq m, 1 \leq j \leq k$. Then $L:=\bigcap_{1 \leq j \leq k} M_{j}=$ $\bigcup_{i \in I} L\left(\boldsymbol{b}_{i}, P\right)$ such that

- $\|L\| \leq(k \cdot m \cdot s+1)^{O(k \cdot n)}$

- for every $\boldsymbol{b}_{i}$ and $1 \leq j \leq k$ there is some $\boldsymbol{d} \geq \mathbf{0}$ such that $\boldsymbol{b}_{i}=\boldsymbol{c}_{j}+Q_{j} \cdot \boldsymbol{d}$, and

- for every $1 \leq j \leq k$ there is some non-negative matrix $R$ such that $P=Q_{j} \cdot R$.

Proof. Consider the following system of linear Diophantine equations $\mathcal{S}$ :

$$
\begin{aligned}
\boldsymbol{c}_{1}+Q_{1} \cdot \boldsymbol{\lambda}_{1} & =\boldsymbol{c}_{2}+Q_{2} \cdot \boldsymbol{\lambda}_{2} \\
\boldsymbol{c}_{2}+Q_{2} \cdot \boldsymbol{\lambda}_{2} & =\boldsymbol{c}_{3}+Q_{3} \cdot \boldsymbol{\lambda}_{3} \\
\vdots & \\
\boldsymbol{c}_{k-1}+Q_{k-1} \cdot \boldsymbol{\lambda}_{k-1} & =\boldsymbol{c}_{k}+Q_{k} \cdot \boldsymbol{\lambda}_{k}
\end{aligned}
$$

The constraint matrix of $\mathcal{S}$ has $k \cdot n$ rows and at most $k \cdot n$ columns, and $\|\mathcal{S}\| \leq 2 s$. According to [10, Prop. 4], we have $\llbracket \mathcal{S} \rrbracket=L(E, S)$ such that $\|L(E, S)\| \leq((k \cdot m+3) \cdot\|\mathcal{S}\|+$ $1)^{k \cdot n}$. For every $1 \leq j \leq k$, let $D_{j}$ and $R_{j}$ be obtained from the projection of $E$ respectively $S$ onto the components corresponding to $\boldsymbol{\lambda}_{j}$. Then as in the proof of [10. Thm. 6], for any $1 \leq j \leq k$ we have

$$
\begin{aligned}
L & =\boldsymbol{c}_{j}+\left\{Q_{j} \cdot \gamma: \gamma \in L\left(D_{j}, R_{j}\right)\right. \\
& =L\left(\boldsymbol{c}_{j}+Q_{j} \cdot D_{j}, Q_{j} \cdot R_{j}\right) .
\end{aligned}
$$

A quick inspection reveals

$$
\begin{aligned}
\left\|Q_{j} \cdot R_{j}\right\| & \leq\left\|\boldsymbol{c}_{j}+Q_{j} \cdot D_{j}\right\| \\
& \leq\left\|\boldsymbol{c}_{j}\right\|+m s((k m+3) \cdot\|\mathcal{S}\|+1)^{k n} \\
& \leq(k \cdot m \cdot s+1)^{O(k \cdot n)}
\end{aligned}
$$




\section{APPENDIX B}

\section{MISSING PROOF FROM SECTION VI}

Correctness proof for Proposition VI.1 We define the subset $A_{\ell}=\left\{a_{1}, \ldots, a_{\ell}\right\}$. Observe that since the $\left[a_{i}\right]$ commute pairwise, we can regard the morphism $\pi_{A_{\ell}}$ as $\pi_{A_{\ell}}: \mathbb{G}(A, I) \rightarrow \mathbb{Z}^{\ell}$ and shorten it to $\pi_{\ell}$. We claim that for words $w \in\left\{b_{1}, \ldots, b_{d}\right\}^{*}$ and $y_{i} \in\left\{a_{1}^{ \pm 1}, \ldots, a_{d}^{ \pm 1}\right\}^{*}$, we have $\left[x y_{1} b_{\ell_{1}}^{-1} y_{2} b_{\ell_{2}}^{-1} \cdots y_{n} b_{\ell_{n}}\right]=1$ if and only if $x=b_{\ell_{n}} \cdots b_{\ell_{1}}$, $\left[y_{1} \cdots y_{n+1}\right]=1$ and $\pi_{\ell_{i}}\left(y_{1} \cdots y_{i}\right)=0$ for every $i \in[1, n]$. Note that the claim immediately implies that $1 \in \mathrm{L}\left(\mathcal{A}, q_{0}, q^{\prime}\right)$ if and only if $(q, 0) \rightarrow_{V}^{*}\left(q^{\prime}, 0\right)$.

For the "if" direction, suppose $x=b_{\ell_{n}} \cdots b_{\ell_{1}}$. $\left[y_{1} \cdots y_{n+1}\right]=1$ and $\pi_{\ell_{i}}\left(y_{1} \cdots y_{i}\right)=0$ for every $i \in[1, n]$. We proceed by induction on $n$. If $n=0$, then the claim is trivial. Let $n \geq 1$. Since the $a_{i}$ commute pairwise, we can write $\left[y_{1}\right]=\left[z z^{\prime}\right]$ with some $z \in\left\{a_{1}^{ \pm 1}, \ldots, a_{\ell_{1}}^{ \pm 1}\right\}^{*}$ and $z^{\prime} \in$ $\left\{a_{\ell_{1}+1}^{ \pm 1}, \ldots, a_{d}^{ \pm 1}\right\}^{*}$. Moreover, $\pi_{\ell_{1}}\left(y_{1}\right)=0$ implies $[z]=1$. Since $b_{\ell_{1}}$ commutes with all the generators $a_{\ell_{1}+1}, \ldots, a_{d}$, we have

$$
\begin{aligned}
& {\left[x y_{1} b_{\ell_{1}}^{-1} y_{2} b_{\ell_{2}}^{-1} \cdots y_{n} b_{\ell_{n}}^{-1} y_{n+1}\right] } \\
= & {\left[x z z^{\prime} b_{\ell_{1}}^{-1} y_{2} b_{\ell_{2}}^{-1} \cdots y_{n} b_{\ell_{n}}^{-1} y_{n+1}\right] } \\
= & {\left[x z b_{\ell_{1}}^{-1} z^{\prime} y_{2} b_{\ell_{2}}^{-1} \cdots y_{n} b_{\ell_{n}}^{-1} y_{n+1}\right] } \\
= & {\left[b_{\ell_{n}} \cdots b_{\ell_{1}} z b_{\ell_{1}}^{-1} z^{\prime} y_{2} b_{\ell_{2}}^{-1} \cdots y_{n} b_{\ell_{n}}^{-1} y_{n+1}\right] } \\
= & {\left[b_{\ell_{n}} \cdots b_{\ell_{2}} z^{\prime} y_{2} b_{\ell_{2}}^{-1} \cdots y_{n} b_{\ell_{n}}^{-1} y_{n+1}\right] . }
\end{aligned}
$$

Note that $[z]=1$ implies $\pi_{\ell_{i}}\left(z^{\prime} y_{2} \cdots y_{i}\right)=$ $\pi_{\ell_{i}}\left(z z^{\prime} y_{2} \cdots y_{i}\right)=\pi_{\ell_{i}}\left(y_{1} \cdots y_{i}\right)=0$ for every $i \in[1, n]$. Therefore, we may invoke induction to conclude that $\left[b_{\ell_{n}} \cdots b_{\ell_{2}} z^{\prime} y_{2} b_{\ell_{2}}^{-1} \cdots y_{n} b_{\ell_{n}}^{-1}\right]=1$ holds and thus $\left[x y_{1} b_{\ell_{1}}^{-1} y_{2} b_{\ell_{2}}^{-1} \cdots y_{n} b_{\ell_{n}}^{-1}\right]=1$.

For the "only if" direction, suppose the word $w=$ $x y_{1} b_{\ell_{1}}^{-1} y_{2} b_{\ell_{2}}^{-1} \cdots y_{n} b_{\ell_{n}}^{-1}$ satisfies $[w]=1$. We again proceed by induction and notice that the claim holds vacuously for $n=0$. Now let $n \geq 1$. First, observe that $[w]=1$ implies $\left[x b_{\ell_{1}}^{-1} b_{\ell_{2}}^{-1} \cdots b_{\ell_{n}}^{-1}\right]=\pi_{B}(w)=1$ and thus $[x]=\left[b_{\ell_{n}} \cdots b_{\ell_{1}}\right]$. Since the $b_{i}$ do not commute with each other, that means $x=b_{\ell_{n}} \cdots b_{\ell_{1}}$. We can find $z \in\left\{a_{1}^{ \pm 1}, \ldots, a_{\ell_{1}}^{ \pm 1}\right\}^{*}$ and $z^{\prime} \in\left\{a_{\ell_{1}+1}^{ \pm 1}, \ldots, a_{d}^{ \pm 1}\right\}^{*}$ such that $\left[y_{1}\right]=\left[z z^{\prime}\right]$. Toward a contradiction, suppose $[z] \neq 1$. Then it is easy to see by induction on the number of applied rewriting steps that any word $w^{\prime} \in\left(A^{ \pm 1}\right)^{*}$ with $\left[w^{\prime}\right]=[w]$ has an infix $b_{\ell_{1}} y b_{\ell_{1}}^{-1}$ with $\pi_{\ell_{1}}(y)=[z] \neq 0$ : We can certainly not apply any cancellation rule for a $b_{i}$; other cancellation rules might be applicable, but preserve this property; and since $b_{\ell_{1}}$ does not commute with $a_{1}, \ldots, a_{\ell_{1}}$, commutation rules preserve this property as well. Therefore, we would have $[w] \neq 1$. Thus, we have shown $[z]=1$.

Observe that

$$
\begin{aligned}
{[w] } & =\left[b_{\ell_{n}} \cdots b_{\ell_{1}} z z^{\prime} b_{\ell_{1}}^{-1} y_{2} b_{\ell_{2}}^{-1} \cdots y_{n} b_{\ell_{n}}^{-1} y_{n+1}\right] \\
& =\left[b_{\ell_{n}} \cdots b_{\ell_{1}} z b_{\ell_{1}}^{-1} z^{\prime} y_{2} b_{\ell_{2}}^{-1} \cdots y_{n} b_{\ell_{n}}^{-1} y_{n+1}\right] \\
& =\left[b_{\ell_{n}} \cdots b_{\ell_{2}} z^{\prime} y_{2} b_{\ell_{2}}^{-1} \cdots y_{n} b_{\ell_{n}}^{-1} y_{n+1}\right] .
\end{aligned}
$$

By induction, we may conclude that $\pi_{\ell_{i}}\left(z^{\prime} y_{2} \cdots y_{i}\right)=1$ for every $i \in[2, n+1]$ and $\left[z^{\prime} y_{2} \cdots y_{n+1}\right]=1$. Finally, observe that since $[z]=1$, we have $\pi_{\ell_{i}}\left(y_{1} \cdots y_{i}\right)=\pi_{\ell_{i}}\left(z z^{\prime} y_{2} \cdots y_{i}\right)=$ $\pi_{\ell_{i}}\left(z^{\prime} y_{2} \cdots y_{i}\right)=0$ and for $i \in[1, n]$ and $\left[y_{1} \cdots y_{n+1}\right]=$ $\left[z z^{\prime} y_{2} \cdots y_{n+1}\right]=1$.

\section{APPENDIX C}

\section{MISSING PROOFS FROM SECTIONVII}

In order to prove Proposition VII.4, we need a simple fact about free products of groups. Since $A=A_{0} \uplus A_{1}$, we can uniquely decompose each $u \in\left(A^{ \pm 1}\right)^{*}$ into maximal factors from $\left(A_{0}^{ \pm 1}\right)^{+} \cup\left(A_{1}^{ \pm 1}\right)^{+}$. In other words, we decompose $u=$ $u_{1} \cdots u_{n}$ with $n \geq 0$ such that $u_{i} \in\left(A_{0}^{ \pm 1}\right)^{+} \cup\left(A_{1}^{ \pm 1}\right)^{+}$for $i \in[1, n]$ and $u_{i} \in\left(A_{s}^{ \pm 1}\right)^{+}$if and only if $u_{i+1} \in\left(A_{1-s}^{ \pm 1}\right)^{+}$for $i \in[1, n-1]$ and $s \in\{0,1\}$. This is the block decomposition of $u$.

Lemma C.1. Let $u \in\left(A^{ \pm 1}\right)^{*}$ and let $u=u_{1} \cdots u_{n}$ be its block decomposition. If $[u]=1$, then $\left[u_{i}\right]=1$ for some $i$.

Proof. Suppose $\left[u_{i}\right] \neq 1$ for every $i \in[1, n]$. By induction on the number of rewriting steps, it is easy to see that every word $u^{\prime}$ with $[u]=\left[u^{\prime}\right]$ has a block decomposition $u^{\prime}=u_{1}^{\prime} \cdots u_{m}^{\prime}$ with $m=n$ and $\left[u_{i}^{\prime}\right]=\left[u_{i}\right]$ for every $i \in[1, n]$. For cancellation rules, that is trivial. Moreover, commutation rules can only rearrange symbols within each block (recall that we assumed that there are no edges between $A_{0}$ and $A_{1}$ ). This implies that $u^{\prime}$ cannot be the empty word and hence $[u] \neq 1$.

Proposition VII.4. For every $p, q \in Q$, we have $\mathrm{L}\left(G_{\mathcal{A}}, \square_{p}^{q}\right)=$ $\mathrm{L}(\mathcal{A}, p, q)$.

Proof. The following notation for runs in valence automata will be convenient: If in a valence $\mathcal{A}$, we have $(p, 1, \varepsilon) \rightarrow_{\mathcal{A}}^{*}$ $(q,[u], v)$, then we write $p \stackrel{u \mid v}{\longrightarrow} q$.

We begin with the inclusion $\mathrm{L}\left(G_{\mathcal{A}}, \square_{p}^{q}\right) \subseteq \mathrm{L}(\mathcal{A}, p, q)$. We prove the inclusion by establishing the following claim: For every $v \in(N \cup \Sigma)^{*}$ with $\square_{p}^{q} \Rightarrow^{k} v$, we have $v_{0} \square_{p_{1}}^{q_{1}} v_{1} \cdots \square_{p_{n}}^{q_{n}} v_{n}$ for some $n \geq 0$ and there are runs

$$
\begin{aligned}
p \stackrel{u_{0} \mid v_{0}}{\longrightarrow} p_{1}, q_{1} \stackrel{u_{1} \mid v_{1}}{\longrightarrow} p_{2}, \ldots, \\
q_{n-1} \stackrel{u_{n-1} \mid v_{n-1}}{\longrightarrow} p_{n}, q_{n} \stackrel{u_{n} \mid v_{n}}{\longrightarrow} q
\end{aligned}
$$

in $\mathcal{A}$ such that $\left[u_{0} \cdots u_{n}\right]=1$. The claim implies that for every $v \in \mathrm{L}\left(G_{\mathcal{A}}, \square_{p}^{q}\right)$, there is a run $p \stackrel{u \mid v}{\longrightarrow} q$ with $[u]=1$, which means $v \in \mathrm{L}(\mathcal{A}, p, q)$. We prove the claim by induction on $k$.

If $k=0$, then $v=\square_{p}^{q}$ and the claim is trivial. Let $k \geq 1$ and $\square_{p}^{q} \Rightarrow^{k-1} v^{\prime} \Rightarrow v$. Then we can write $v^{\prime}=v_{0} \square_{p_{1}}^{q_{1}} v_{1} \cdots \square_{p_{n}}^{q_{n}} v_{n}$ for some $n \geq 0$ and have runs as in Eq. (15). This means $v$ is obtained from $v^{\prime}$ by replacing a nonterminal, say $\square_{p_{i}}^{q_{i}}$, by some word $v^{\prime \prime} \in \mathrm{L}\left(\mathcal{A}_{j}, p_{i}, q_{i}\right)$ for some $j \in\{0,1\}$. By definition of $\mathcal{A}_{j}$, we have $v^{\prime \prime}=v_{0}^{\prime \prime} \square_{p_{1}^{\prime}}^{q_{1}^{\prime}} v_{1}^{\prime \prime} \cdots \square_{p_{m}^{\prime}}^{q_{m}^{\prime}} v_{m}^{\prime \prime}$ and runs

$$
\begin{aligned}
p_{i} \stackrel{u_{0}^{\prime \prime} \mid v_{0}^{\prime \prime}}{\longrightarrow} p_{1}^{\prime \prime}, & q_{1}^{\prime \prime} \stackrel{u_{1}^{\prime \prime} \mid v_{1}^{\prime \prime}}{\longrightarrow} p_{2}^{\prime \prime}, \ldots, \\
& q_{m-1}^{\prime \prime} \stackrel{u_{m-1}^{\prime \prime} \mid v_{m-1}^{\prime \prime}}{\longrightarrow} p_{m}^{\prime \prime}, q_{m}^{\prime \prime} \stackrel{u_{m}^{\prime \prime} \mid v_{m}^{\prime \prime}}{\longrightarrow} q_{i}
\end{aligned}
$$


such that $\left[u_{0}^{\prime \prime} \cdots u_{m}^{\prime \prime}\right]=1$. By inserting the runs in Eq. 16 into Eq. 15 between $p_{i}$ and $q_{i}$, we obtain runs

$$
\begin{gathered}
p \stackrel{u_{0} \mid v_{0}}{\longrightarrow} p_{1}, q_{1} \stackrel{u_{1} \mid v_{1}}{\longrightarrow} p_{2}, \ldots, q_{i-2} \stackrel{u_{i-2} \mid v_{i-2}}{\longrightarrow} p_{i-1}, \\
q_{i-1} \stackrel{u_{i-1} u_{0}^{\prime \prime} \mid v_{i-1} v_{0}^{\prime \prime}}{\longrightarrow} p_{1}^{\prime \prime}, q_{1}^{\prime \prime} \stackrel{u_{1}^{\prime \prime} \mid v_{1}^{\prime \prime}}{\longrightarrow} p_{2}^{\prime \prime}, \ldots, \\
q_{m-1}^{\prime \prime} \stackrel{u_{m-1}^{\prime \prime} \mid v_{m-1}^{\prime \prime}}{\longrightarrow} p_{m}^{\prime \prime}, q_{m}^{\prime \prime} \stackrel{u_{m}^{\prime \prime} u_{i} \mid v_{m}^{\prime \prime} v_{i}}{\longrightarrow} p_{i+1}, \\
q_{i+1} \stackrel{u_{i+1} \mid v_{i+1}}{\longrightarrow} p_{i+1}, \ldots, \\
q_{n-1} \stackrel{u_{n-1} \mid v_{n-1}}{\longrightarrow} p_{n}, q_{n} \stackrel{u_{n} \mid v_{n}}{\longrightarrow} q .
\end{gathered}
$$

Since

$$
\begin{array}{r}
v=v_{0} \square_{p_{1}}^{q_{1}} v_{1} \cdots \square_{p_{i-1}}^{q_{i-1}} v_{i-1}\left(v_{0}^{\prime \prime} \square_{p_{1}^{\prime \prime}}^{q_{1}^{\prime \prime}} v_{1}^{\prime \prime} \cdots \square_{p_{m}^{\prime \prime}}^{q_{m}^{\prime \prime}} v_{m}^{\prime \prime}\right) \\
v_{i} \square_{p_{i+1}}^{q_{i+1}} \cdots v_{n-1} \square_{p_{n}}^{q_{n}} v_{n},
\end{array}
$$

this proves the claim for $v$.

Let us now prove the inclusion $\mathrm{L}(\mathcal{A}, p, q) \subseteq \mathrm{L}\left(G_{\mathcal{A}}, \square_{p}^{q}\right)$. We claim that for every sequence of runs as in Eq. 15 in $\mathcal{A}$ with $\left[u_{0} \cdots u_{n}\right]=1$, we have $\square_{p}^{q} \Rightarrow^{*} v_{0} \square_{p_{1}}^{q_{1}} v_{1} \cdots \square_{p_{n}}^{q_{n}} v_{n}$. Note that this establishes the inclusion: For each $v \in \mathrm{L}(\mathcal{A}, p, q)$, there is a run $p \stackrel{u \mid v}{\longrightarrow} q$ with $[u]=1$. Thus, the claim implies $\square_{p}^{q} \Rightarrow^{*} v$, which means $v \in \mathrm{L}\left(G_{\mathcal{A}}, \square_{p}^{q}\right)$ because $v \in \Sigma^{*}$.

We proceed by induction on the number of blocks in $u=$ $u_{0} \cdots u_{n}$. According to Lemma C.1, the block decomposition of the word $u_{0} \cdots u_{n}$ contains some block $w$ with $[w]=1$. Now $w$ can occur within a single factor $u_{i}$ or span several factors. We only consider the latter case, the former can be treated analogously.

Hence, we can find indices $i<j$ and a decomposition $u_{i}=$ $u_{i}^{\prime} u_{i}^{\prime \prime}$ and $u_{j}=u_{j}^{\prime} u_{j}^{\prime \prime}$ such that $w=u_{i}^{\prime \prime} u_{i+1} \cdots u_{j-1} u_{j}^{\prime}$. Since $\mathcal{A}$ reads at most one generator from $A^{ \pm 1}$ in each edge, this decomposition induces a decomposition of the runs $q_{i} \stackrel{u_{i} \mid v_{i}}{\longrightarrow}$ $p_{i+1}$ and $q_{j} \stackrel{u_{j} \mid v_{j}}{\longrightarrow} p_{j+1}$ into

$$
q_{i} \stackrel{u_{i}^{\prime} \mid v_{i}^{\prime}}{\longrightarrow} p^{\prime} \stackrel{u_{i}^{\prime \prime} \mid v_{i}^{\prime \prime}}{\longrightarrow} p_{i+1} \text { and } q_{j} \stackrel{u_{j}^{\prime} \mid v_{j}^{\prime}}{\longrightarrow} q^{\prime} \stackrel{u_{j}^{\prime \prime} \mid v_{j}^{\prime \prime}}{\longrightarrow} p_{j+1} .
$$

Consider the sequence of runs that is obtained from Eq. 15 by replacing $q_{i} \stackrel{u_{i} \mid v_{i}}{\longrightarrow} p_{i+1}$ by $q_{i} \stackrel{u_{i}^{\prime} \mid v_{i}^{\prime}}{\longrightarrow} p^{\prime}$ and replacing $q_{j} \stackrel{u_{j} \mid v_{j}}{\longrightarrow} p_{j+1}$ by $q^{\prime} \stackrel{u_{j}^{\prime \prime} \mid v_{j}^{\prime \prime}}{\longrightarrow} p_{j+1}$. Then the word $u_{0} \cdots u_{i-1} u_{i}^{\prime} u_{j}^{\prime \prime} u_{j+1} \cdots u_{n}$ has one fewer block than $u_{0} \cdots u_{n}$. Hence, by induction, the word

$v^{\prime}=v_{0} \square_{p_{1}}^{q_{1}} v_{1} \cdots \square_{p_{i-1}}^{q_{i-1}} v_{i-1} \square_{p_{i}}^{q_{i}} v_{i}^{\prime} \square_{p^{\prime}}^{q^{\prime}} v_{j}^{\prime \prime} \square_{p_{j+1}}^{q_{j+1}} v_{j+1} \cdots \square_{p_{n}}^{q_{n}} v_{n}$ verifies $\square_{p}^{q} \Rightarrow{ }^{*} v^{\prime}$. On the other hand, since $w$ was chosen to be a block, we have $u_{i}^{\prime \prime} u_{i+1} \cdots u_{j-1} u_{j}^{\prime}=w \in\left(A_{s}^{ \pm 1}\right)^{+}$for some $s \in\{0,1\}$ and therefore runs

$$
\begin{aligned}
p^{\prime} \stackrel{u_{i}^{\prime \prime} \mid v_{i}^{\prime \prime}}{\longrightarrow} \mathcal{A}_{s} p_{i+1}, q_{i+1} \stackrel{u_{i+1} \mid v_{i+1}}{\longrightarrow} \mathcal{A}_{s} p_{i+2}, \ldots, \\
q_{j-1} \stackrel{u_{j-1} \mid v_{j-1}}{\longrightarrow} \mathcal{A}_{s} p_{j}, q_{j} \stackrel{u_{j}^{\prime} \mid v_{j}^{\prime}}{\longrightarrow} \mathcal{A}_{s} q^{\prime}
\end{aligned}
$$

in $\mathcal{A}_{s}$. By construction of $\mathcal{A}_{s}$, this means we have $v^{\prime \prime}=$ $v_{i}^{\prime \prime} \square_{p_{i+1}}^{q_{i+1}} v_{i+1} \cdots \square_{p_{j-1}}^{q_{j-1}} v_{j-1} \square_{p_{j}}^{q_{j}} v_{j}^{\prime} \in \mathrm{L}\left(\mathcal{A}_{s}, p^{\prime}, q^{\prime}\right)$. Therefore, replacing the occurrence of $\square_{p^{\prime}}^{q^{\prime}}$ in $v^{\prime}$ by $v^{\prime \prime}$ yields $v$, hence $\square_{p}^{q} \Rightarrow{ }^{*} v^{\prime} \Rightarrow v$.

\section{A. Analysis}

We now analyze our translation described in Steps I to III On one hand, we show that the translation maps RatMP ${ }_{k}^{\text {tf }}$ to formulas of star-height $\leq k$. On the other hand, we show that it runs in polynomial time.

Concerning the star-height, suppose we are given a transitive forest $(A, I)$ with $\beta(A, I)=k$. Notice that our recursive algorithm introduces a star only in step Step II Compared to the formulas of the automata $\mathcal{A}_{0}$ and $\mathcal{A}_{1}$, it raises the star height by one. According to the definition of the branching number, this directly implies that the resulting $\exists \mathrm{PA}^{*}$ formula has star-height $k$.

Let us now show that the construction runs in polynomial time. It is clear from the construction that computing the formulas takes time linear in the size of the formulas. Hence, it suffices to show that they are polynomial in size. Let $\Phi_{\mathcal{A}}$ denote the formula computed for each automaton $\mathcal{A}$. Now suppose the input automaton $\mathcal{A}$ has $n$ states and $m$ edges. An inspection of Steps II to III reveals that there is a polynomial $p(x, y)$ such that:

1) If $(A, I)$ has a universal vertex, then $\left|\Phi_{\mathcal{A}}\right| \leq\left|\Phi_{\mathcal{A}^{\prime}}\right|+$ $p(n, m)$.

2) If $(A, I)$ is a disjoint union, then $\left|\Phi_{\mathcal{A}}\right| \leq\left|\Phi_{\mathcal{A}_{0}}\right|+\left|\Phi_{\mathcal{A}_{1}}\right|+$ $p(n, m)$.

3) If $(A, I)$ is empty, then $\left|\Phi_{\mathcal{A}}\right| \leq p(n, m)$.

Note that the length of the formulas may depend on the size of the alphabet, but that is bounded by the number $m$ of edges. Of course, we may assume that $p$ has only positive coefficients.

Let us show by induction on $|A|$ that $\left|\Phi_{\mathcal{A}}\right| \leq|A|^{2} p(n, m+$ $\left.|A| n^{2}\right)$ for every $\mathcal{A}$. If $(A, I)$ is empty, then this is clear. If $(A, I)$ has a universal vertex, then we construct $\mathcal{A}^{\prime}$ over $\left(A^{\prime}, I^{\prime}\right)$, where $\mathcal{A}^{\prime}$ has $n$ states and $m$ edges and $\left|A^{\prime}\right|=|A|-1$. Therefore:

$$
\begin{aligned}
\left|\Phi_{\mathcal{A}}\right| & \leq\left|\Phi_{\mathcal{A}^{\prime}}\right|+p(|A|, n, m) \\
& \leq(|A|-1)^{2} p\left(n, m+|A| n^{2}\right)+p(n, m) \\
& \leq|A|^{2} p\left(n, m+|A| n^{2}\right) .
\end{aligned}
$$

Now suppose $(A, I)$ is a disjoint union of $\left(A_{0}, I_{0}\right)$ and $\left(A_{1}, I_{1}\right)$. Then we construct $\mathcal{A}_{0}$ and $\mathcal{A}_{1}$. They each have $n$ states and $m+n^{2}$ edges, because we add the edges labeled $\square_{p}^{q}$ for each $p, q \in Q$. Moreover, we have $\left|A_{0}\right|,\left|A_{1}\right|<|A|$ and thus by induction

$\left|\Phi_{\mathcal{A}_{i}}\right| \leq\left|A_{i}\right|^{2} p\left(n, m+n^{2}+\left|A_{i}\right| n^{2}\right) \leq\left|A_{i}\right|^{2} p\left(n, m+|A| n^{2}\right)$.

That implies

$$
\begin{aligned}
\left|\Phi_{\mathcal{A}}\right| & \leq\left|\Phi_{\mathcal{A}_{0}}\right|+\left|\Phi_{\mathcal{A}_{1}}\right|+p(n, m) \\
& \leq\left(\left|A_{0}\right|^{2}+\left|A_{1}\right|^{2}\right) p\left(n, m+|A| n^{2}\right)+p(n, m) \\
& \leq\left(\left|A_{0}\right|^{2}+\left|A_{1}\right|^{2}+1\right) p\left(n, m+|A| n^{2}\right) \\
& \leq|A|^{2} p\left(n, m+|A| n^{2}\right) .
\end{aligned}
$$

Thus, the resulting formulas have polynomial size. 